\title{
Automatizability and Simple Stochastic Games
}

\author{
Lei Huang * \\ University of Toronto \\ leih@cs.toronto.edu
}

\author{
Toniann Pitassi* \\ University of Toronto \\ toni@cs.toronto.edu
}

April 24, 2011

\begin{abstract}
The complexity of simple stochastic games (SSGs) has been open since they were defined by Condon in 1992. Despite intensive effort, the complexity of this problem is still unresolved. In this paper, building on the results of [4], we establish a connection between the complexity of SSGs and the complexity of an important problem in proof complexity-the proof search problem for low depth Frege systems. We prove that if depth-3 Frege systems are weakly automatizable, then SSGs are solvable in polynomial-time. Moreover we identify a natural combinatorial principle, which is a version of the well-known Graph Ordering Principle (GOP), that we call the integer-valued GOP (IGOP). This principle states that for any graph $G$ with nonnegative integer weights associated with each node, there exists a locally maximal vertex (a vertex whose weight is at least as large as its neighbors). We prove that if depth-2 Frege plus IGOP is weakly automatizable, then SSG is in P.
\end{abstract}

\footnotetext{
${ }^{*}$ Supported by NSERC.
} 


\section{Introduction}

In a groundbreaking paper from 1992, Condon defined the family of simple stochastic games (SSGs) [14]. A simple stochastic game is a directed graph with four types of vertices: min nodes, max nodes, average nodes, and two sinks, a 0 -sink and a 1-sink. The game is played by two players, Max and Min, from a given start node. The goal of Max is to reach the 1-sink, while the goal of Min is to reach the 0 -sink, or to continue the game indefinitely. The value of the game is the probability that Max wins, assuming that both players play optimally.

A fascinating open question is determining the complexity of finding the value of a game, or equivalently, the complexity of finding an optimal strategy. From a practical point of view, stochastic games are used to model a variety of problems in software verification and controller optimization, and from a theoretical point of view, the SSG problem is fundamental since an efficient algorithm for it will imply algorithms for a host of other problems. Indeed, it is known to be polynomial-time equivalent to many other computational problems such as the generalized linear complementarity problem, and the minimum stable circuit problem. Many other game-theoretic problems, such as mean payoff games, discounted payoff games, and parity games, all reduce to SSGs [2]. Furthermore, SSG is also the complete problem for the class AUC-SPACE $(\log n)$ of logspace bounded alternating random turing machines [14]. A polynomial time algorithm solving the SSG problem would imply that adding random nodes to logspace bounded alternating turing machines does not grant any additional computational power.

Despite considerable effort over the last decades, it remains a longstanding open problem to determine the complexity of SSGs. Condon [14, 15] proved that the SSG decision problem, "does the max player have a strategy ensuring at least $\frac{1}{2}$ probability of winning", is in both NP and coNP. This makes SSGs one of the few combinatorial problems known to be in $N P \cap \operatorname{coNP}$ and suggests that it is very unlikely to be NP-complete. Another natural avenue for obtaining a hardness result for SSGs are the complexity classes associated with total functions in NP, such as PLS and PPAD. In fact, recently the complexity of the Nash Equilibrium problem was resolved in a celebrated sequence of papers, culminating in a proof of the PPAD-completeness of Nash $[12,17]$ Unfortunately, such a hardness result for SSGs is unlikely, as it was shown to lie in both PLS and PPAD [6].

The SSG problem has also been studied extensively from an algorithmic point of view [15, 19, 25, 23, 20, 5, 22]. Many restrictions such as allowing only two of three types of vertices, or to a constant number of random nodes, are known to have polynomial time algorithms [15, 19]. However, the current best algorithm for the full game is a $O\left(2^{\sqrt{n}}\right)$ time randomized algorithm $[23,20]$, and a $O\left(n \cdot\left|V_{r}\right| !\right)$ time deterministic algorithm [19], where $V_{r}$ is the set of average nodes.

A seemingly unrelated, but also longstanding and important problem is to determine whether proofs in standard proof systems can be found efficiently. A proof system $\mathcal{P}$ is automatizable if there exists an algorithm that takes as input a tautology $f$, and outputs a $\mathcal{P}$-proof of $f$, and such that the runtime is polynomial in the size of the shortest $\mathcal{P}$-proof of $f$. $\mathcal{P}$ is weakly automatizable if there is an algorithm that on input $f$ and a number $r$ in unary, can distinguish the case where $f$ is not a tautology from the case where $f$ has a $\mathcal{P}$-proof of size at most $r$. It is known that $\mathcal{P}$ is weakly-automatizable if and only if there is an automatizable proof system that simulates $\mathcal{P}$ [3].

The question of whether standard proof systems are automatizable is a fundamental question in logic and automated theorem proving [10]. Following [21], it was shown that Frege systems are not weakly automatizable under a widely believed cryptographic assumption - that the Diffie Hellman (DH) problem (and hence factoring) is hard to compute. However, despite considerable effort, the weak automatizability question for low-depth Frege systems is unresolved. [7] showed 
that $A C_{0}^{k}$-Frege is not weakly automatizable under the assumption that the $\mathrm{DH}$ problem can be solved in time $\exp \left(n^{c / k}\right)$, where $c>2$. The best algorithm for DH runs in time $\exp \left(n^{1 / 2}\right)$, and moreover the number field sieve is conjectured to solve DH in time $\exp \left(n^{1 / 3}\right)$; thus for small $k(k$ less than 5 or 6 ), the weak automatizability of depth-k Frege is unresolved. Even for depth-1 Frege (i.e. resolution), there is no clear evidence for or against weak automatizability, despite results in both directions $[1,13]$.

Results and Related Work. In a recent paper, Atserias and Maneva made an important new link between automatizability and game theory by proving that solving mean payoff games (MPGs) is reducible to the weak automatizability of depth-2 Frege systems and to feasible interpolation of depth-3 Frege systems[4]. In this paper, we prove that if depth-3 Frege systems are weakly automatizable, then simple stochastic games are solvable in polynomial time, thus establishing a link between SSGs and an important open problem in proof complexity.

Mean payoff games can be viewed as a special case of simple stochastic games, but depth-2 Frege systems can also be viewed as a special case of depth-3 Frege thus the results cannot be directly compared. However, the increase in depth suggests an approach to pinpointing a difference between MPGs and SSGs, an interesting open problem in its own right. Moreover, the only part of our proof that is not contained in depth-2 is in the proof of a natural combinatorial property about graphs that we will call the Integer-Valued Graph Ordering Principle (IGOP). IGOP states informally that in any finite undirected graph where all nodes are labelled by integers, there exists a vertex whose value is at least as large as its neighbors. This principle is expressible as a CNF formula, and we actually prove that if depth-2 Frege, augmented with IGOP, is weakly automatizable, then SSGs are in $\mathrm{P}$. This raises the very interesting question as to the exact proof theoretic strength required to prove IGOP: If it has a polynomial size depth-2 Frege proof, then our result subsumes that of [4]. On the other hand, if not, then we have found a natural CNF formula separating depth-2 from depth-3 Frege, and furthermore, expose an essential difference between mean payoff games and simple stochastic games.

Our proof builds on [4] in that we use and further develop very low depth circuits that they invent for performing addition of a constant number of integers. However, at a high level our proofs are very different. [4] goes through an intermediate transformation to the Max Atom Problem that appears to hold only for mean payoff games. In contrast our proof strategy is much more generalizable. The main idea relies on the unique fixed point property of SSGs - ie SSGs can be characterized as a fixed point computation where the unique fixed point indicates the winner. This property is shared by a variety of other problems, including the more general class of stochastic games defined by Shapley [24].

Proof Overview. The basic idea behind our proof is to study the complexity of stopping games, which are polynomial-time equivalent to general SSGs. In a stopping game, the optimal solution is also the only solution to a set of local optimality conditions. We formalize the formulas, $\operatorname{MinWin}(G)$ (and $\operatorname{MaxWin}(G)$ ) expressing that there is a locally optimal strategy with value less than or equal to one half (or greater than one half). Since the locally optimal solution is always unique for stopping games, the formula $F(G)=\operatorname{MinWin}(G) \wedge \operatorname{Max} \operatorname{Win}(G)$ is unsatisfiable. The bulk of our argument is thus to show that $F(G)$, has an efficient depth-3 Frege refutation. Then if depth-3 Frege has feasible interpolation, the interpolant for $F$ will return whether MinWin $(G)$ or $\operatorname{Max} \operatorname{Win}(G)$ is unsatisfiable, thus revealing the winner of the game. Since weak automatizability implies interpolation, it immediately follows that weak automatizability of depth-3 Frege implies that SSG is in $P$. The technical difficulty is to prove that for any stopping game $G$, the locally 
optimal solution is unique. This involves a very careful analysis of low depth circuits for performing arithmetic on sets of numbers, as well as very efficient reasoning about these arithmetic formulas.

\section{Definitions}

\subsection{Simple Stochastic Games}

In a simple stochastic game (SSG) two players take turns moving a pebble along a directed graph $G:(V, E)$ with two terminal positions, a 0 -sink and a 1 -sink. Conventionally the game also has a unique source node from which gameplay starts. All non terminal nodes of $G$ are partitioned into max nodes, min nodes, and random nodes. From a max (min) nodes, Max (Min) chooses the out-edge the pebble takes next. At a random node, the successor node is chosen by chance. We will consider only binary SSGs where every node has out degree two and random nodes choose each out-edge with probability $\frac{1}{2}$. All SSGs have such a binary equivalent.

In a given play, the max player wins if the pebble reaches the 1-sink and the min player wins otherwise (either by reaching the 0 -sink or continuing play indefinitely). Let $\sigma$ be a strategy for Max, $\sigma: V^{*} \rightarrow V$ mapping paths in a play to a legal successor node, and $\tau$ be a strategy for Min. The value of a node under $\sigma$ and $\tau, v_{\sigma, \tau}(i)$, is defined to be the probability that the game ends at 1 -sink if the players use strategies $\sigma, \tau$ and the pebble starts at node $i$. The optimal value $v_{\text {opt }}(i)$ of a node is the minimax over all strategies

$$
v_{\text {opt }}(i)=\max _{\sigma} \min _{\tau} v_{\sigma, \tau}(i)=\min _{\tau} \max _{\sigma} v_{\sigma, \tau}(i)
$$

The value of the game is $v_{\text {opt }}(0)$ where node 0 is the start node.

Fact 1 (Shapley, 53) For every SSG there exists pure positional strategies $\sigma$ and $\tau$ that achieve $v_{\text {opt }}(i)$ at every node $i$.

Shapley proved that in any SSG there always exist optimal strategies that are deterministic and memoryless [24]. Thus a strategy can be thought of as simply a mapping $V \rightarrow V$ from the set of Max (or Min) nodes to their children and optimal strategies are ergodic, they are optimal regardless of which vertex is the start vertex. The SSG-value problem is to determine whether a game $G$ has optimal value $>\frac{1}{2}$.

Fact 2 (Condon, 92) The optimal values of any n node $S S G$ can be written as $\frac{a}{b}$ where $a, b \in \mathbb{N}$, $b=O\left(2^{n}\right)$

Proof Sketch: Once a game has been restricted to any pair of strategies $\sigma, \tau$ the game reduces to a Markov process. The values under the pair of strategies satisfy $2(I-Q) \vec{v}_{\sigma, \tau}=2 \vec{b}$ where all the entries of $Q$ and $\vec{b}$ are in $\left\{0,1, \frac{1}{2}\right\}$. The matrix $Q$ consists of exactly the transition probabilities between the nodes, $Q_{i, j}=\frac{1}{2}$ if $i$ is a random node, 1 if $i$ is a $\max (\min )$ node and $\sigma(i)=j(\tau(i)=j)$, and 0 otherwise. Using Cramer's rule, all entries of $\vec{v}$ are integers over denominator $\operatorname{det}(2(I-Q))$ which an integer $O\left(2^{n}\right)$.

Fact 3 (Condon, 92) The optimal strategies of an SSG are locally greedy. $\sigma$ is an optimal strategy for the max player iff $\sigma(i)=\operatorname{argmax}\left(v_{\text {opt }}(j), v_{\text {opt }}(k)\right)$ for all max nodes $i$ with children $j$ and $k$, and likewise for the min player. Likewise $\tau$ is an optimal strategy for min iff $\tau(i)=\operatorname{argmax}\left(v_{\text {opt }}(j), v_{\text {opt }}(k)\right)$ for all min nodes $i$ with children $j$ and $k$. 
Definition 4 For a simple stochastic game $G$ we define the associated function $I_{G}:\{0,1\}^{n} \rightarrow$ $\{0,1\}^{n}$ as follows.

$$
I_{G}(\vec{x})(i)= \begin{cases}\max \{\vec{x}(j), \vec{x}(k)\} & \text { if } i \text { is a max vertex with successors } j, k \\ \min \{\vec{x}(j), \vec{x}(k)\} & \text { if } i \text { is a min vertex with successors } j, k \\ \frac{1}{2} \vec{x}(j)+\frac{1}{2} \vec{x}(k) & \text { if } i \text { is a average vertex with successors } j, k \\ 0 / 1 & \text { if } i \text { is the zero/one sink }\end{cases}
$$

The vector $\vec{x}$ is stable for $\mathrm{G}$ if for all $i, I_{G}(\vec{x})(i)=\vec{x}(i)$. It follows from Fact 2 that if $\vec{v}$ is the vector of optimal values, $\vec{v}(i)=v_{\text {opt }}(i)$ for all $i$, then $\vec{v}$ is stable for $\mathrm{G}$. In general, there can be more than one stable vector as in Fig. 1.

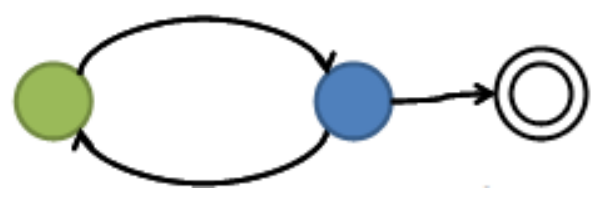

Figure 1: The leftmost node belongs to max, the middle to min, and the rightmost node is the 1 -sink. Both $(1,1,1)$ and $(0,0,1)$ are stable but clearly $(0,0,1)$ is the only correct solution since the min player can force an infinite play.

However, for every game G, there is a corresponding SSG, $G^{\prime}$ such that $G^{\prime}$ has a unique stable vector and moreover, $G$ has optimal value greater than $1 / 2$ if and only if $G^{\prime}$ has optimal value greater than $1 / 2,[14,24]$.

Definition 5 Let $G$ be an $n$ node SSG. The m-stopping game $G^{\prime}$ corresponding to $\mathrm{G}$ is a SSG with $n+m n$ nodes constructed so that any play eventually halts at a terminal node. In other words, no strategies $\sigma, \tau$ can disconnect a node from the terminals. For each vertex $i$ in the original graph of $G, G^{\prime}$ has a network of $m$ average nodes $e_{1 i}, \ldots, e_{m i}$ structured so that $e_{1 i}$ has children $i$ and the 0 -sink, and $e_{j i}$ has children $i$ and $e_{j-1, i}$ when $j>1$. (See Fig. 2 below.) Every in-edge to $i$ in $G$ is replaced by an in-edge to $e_{m i}$ in $G^{\prime}$.

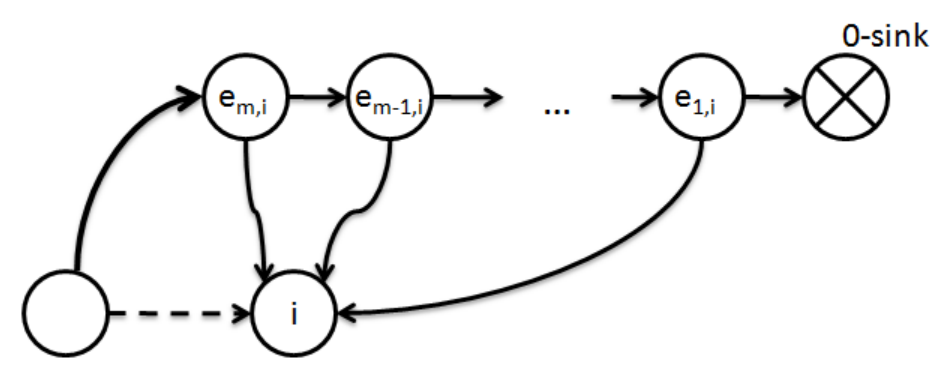

Figure 2: Average node network added to every node $i$ of original game $G$.

Theorem 6 (Condon '92, Shapley 53') If $G$ is an $m$-stopping game (for any $m$ ), then $G$ has a unique stable vector $\vec{x}$.

Theorem 6 implies that finding the optimal values for a stopping game $G$ is equivalent to finding a stable vector for $I_{G^{\prime}}$. This characterization of optimal values will be very useful to us since it 
allows us to express the statement " $\vec{v}$ is the vector of optimal values" in terms of very low level equalities and sums.

Theorem 7 (Condon '92, Shapley 53') For every $G$ there exists a constant $c$ such that the cnstopping game $G^{\prime}$ has value $>\frac{1}{2}$ if and only iff $G$ has value $>\frac{1}{2}$

Proof Sketch: It is easy to show that the optimal values for any stopping game $G^{\prime}$ are always lower than the optimal values in the original $G^{\prime}$. Intuitively, the additional random nodes always increase the probability of reaching the zero sink. Thus if the value of $G^{\prime}>\frac{1}{2}$ then maxthe value of $G>\frac{1}{2}$ also.

From Fact 2 we know that the value of any SSG is not only rational but is also discretized. If $G$ has value $>\frac{1}{2}$, then it actually has value $\geq \frac{1}{2}+\delta$ for some small $\delta=\frac{1}{O\left(2^{n}\right)}$. To prove the converse, it suffices to show that the values of $G$ and $G^{\prime}$ differ by less than $\delta$. As the construction of stopping games adds only the random nodes, any pair of strategies $\sigma^{\prime} \tau^{\prime}$ for $G^{\prime}$ can be restricted to strategies for $G, \sigma$ and $\tau$. For any $G$ there exists a constant $c$ such that if $G^{\prime}$ is the $c n$-stopping game corresponding to $G$ then $\max \left|v_{\sigma, \tau}(i)-v_{\sigma^{\prime}, \tau^{\prime}}^{\prime}(i)\right|<\delta$ [14]. So in particular if $\sigma$ and $\tau$ are optimal strategies then $\left|v_{\text {opt }}(0)-v_{\text {opt }}^{\prime}(0)\right|<\delta$.

\section{$2.2 \quad$ Formulas and Proof Systems}

We will work with the propositional sequent calculus, PK.

In fundamental work of Cook and Reckhow [16], many reasonable formulations of Frege systems were studied and shown to be polynomially equivalent; we work with PK for convenience, but any other Frege system will do.

Each line in a PK proof is a sequent of the form $A_{1}, \ldots, A_{k} \rightarrow B_{1}, \ldots, B_{m}$ where $\rightarrow$ is a new symbol, and $A_{i}, B_{j}$ are formulas. The intended meaning is that the conjunction of the $A_{i}$ 's implies the disjunction of the $B_{j}$ 's. A PK proof of a formula $f$ is a sequence of sequents, such that each sequent is either an instance of the axiom $A \rightarrow A$, or follows from previous sequents from one of the inference rules, and such that the final sequent is $\rightarrow f$.

The rules of PK are of three types: (i) the structural rules, (ii) the logical rules, and (iii) the cut rule. The structural rules are weakening (formulas can always be added to the left or to the right), contraction (two copies of the same formula can be replaced by one) and permutation (formulas in a sequent can be reordered). The final rule is the cut rule, which allows us to derive $\Gamma \longrightarrow \Delta$ from $A, \Gamma \longrightarrow \Delta$ and $\Gamma \longrightarrow A, \Delta$. The logical rules, shown below, allow us to introduce each connective on both the left side and the right side.

1. (Negation Left, $\neg \mathbf{L})$ From $\Gamma \longrightarrow A, \Delta$, derive $\neg A, \Gamma \longrightarrow \Delta$.

2. (Negation Right, $\neg \mathbf{R}$ ) From $A, \Gamma \longrightarrow \Delta$, derive $\Gamma \longrightarrow \neg A, \Delta$.

3. (And Left, $\wedge \mathbf{L}$ ) From $A, B, \Gamma \longrightarrow \Delta$, derive $A \wedge B, \Gamma \longrightarrow \Delta$.

4. (And Right, $\wedge \mathbf{R}$ ) From $\Gamma \longrightarrow A, \Delta$ and $\Gamma \longrightarrow B, \Delta$, derive $\Gamma \longrightarrow A \wedge B, \Delta$.

5. (Or Left, $\vee \mathbf{L})$ From $A, \Gamma \longrightarrow \Delta$ and $B, \Gamma \longrightarrow \Delta$, derive $A \vee B, \Gamma \longrightarrow \Delta$.

6. (Or Right, $\vee \mathbf{R})$ From $\Gamma \longrightarrow A, B, \Delta$ derive $\Gamma \longrightarrow A \vee B, \Delta$. 
The size of a PK proof is the sum of the sizes of all formulas occurring in the proof. The depth of a formula is the number of alternations of OR and ANDs. (Thus the depth of a CNF or DNF formula is two.) We will think of a formula of depth $k$ as an unbounded fanin formula. A formula is $\Sigma_{k}$ if it has depth $k$, where the top (unbounded fanin) connective is OR; a formula is $\Pi_{k}$ if it has depth $k$ and the top (unbounded fanin) connective is AND. A formula is $\Sigma_{k}^{+}\left(\Pi_{k}^{+}\right)$if it has depth $k+1$, where the top connective is OR (AND), and the bottom connective has constant size; a formula is $\Delta_{k}^{+}$if it can be written both as a $\Sigma_{k}^{+}$and as a $\Pi_{k}^{+}$formula. A depth- $k$ PK proof is a PK proof where every formula in the proof has depth at most $k$. Similarly we can define $\Sigma_{k}, \Pi_{k}, \Sigma_{k}^{+}$, $\Pi_{k}^{+}, \Delta_{k}^{+}$PK proofs. It is important to note that depth- $k$ PK proofs are the same as depth- $k+1$ Frege proofs in other non-sequent style axiomatizations. this is because every line in a PK proof is a sequence of formulas, and thus we have an implicit unbounded-fanin OR gate (for free) at the top. We will present PK proofs where every formula in the proof is a $\Delta_{k}^{+}$formula, for some $k$. Such proofs translate into depth $k-1 \mathrm{PK}$ proofs, or equivalently, into depth $k$ Frege proofs (in non sequent-style proof systems).

Definition 8 A proof system $\mathcal{P}$ is automatizable if there exists an algorithm $A$ such that for all unsatisfiable formulas $f A(f)$ returns a $\mathcal{P}$-proof of $f$, and the runtime of $A$ on $f$ is polynomial in the size of the smallest $\mathcal{P}$-proof of $f . \mathcal{P}$ is weakly automatizable if there exists a proof system that polynomially simulates $\mathcal{P}$ and that is automatizable.

Definition 9 Let $F=A(\vec{x}, \vec{z}) \wedge B(\vec{y}, \vec{z})$ be an unsatisfiable formula. An algorithm $C$ is an interpolant for $F$ if for all $\alpha \in\{0,1\}^{|\vec{z}|}, C(\alpha)=1$ implies $A(\vec{x}, \alpha)$ is unsatisfiable, and $C(\alpha)=0$ implies $B(\vec{y}, \alpha)$ is unsatisfiable. A proof system $\mathcal{P}$ admits feasible interpolation if for all unsatisfiable formulas $F=A(\vec{x}, \vec{z}) \wedge B(\vec{y}, \vec{z})$, there exists an algorithm $C$ that is an interpolant for $F$, and the runtime of $C$ is polynomial in the size of the shortest $\mathcal{P}$-proof of $F$.

It was observed in [8] that feasible interpolation and automatizability are closely connected. For any proof system $\mathcal{P}$, if $\mathcal{P}$ is weakly automatizable then $\mathcal{P}$ has feasible interpolation. and the converse direction also holds proved that $\mathcal{P}$ is sufficiently strong. As mentioned in the introduction, under cryptographic assumptions, it has been shown that Frege systems are not automatizable [8]. This work was later scaled down to $A C_{d}^{0}$-Frege systems to show that under much stronger cryptographic assumptions, there exists a constant $d$ such that $A C_{d}^{0}$-Frege systems are also not automatizable [7] However, for small values of $d$ ( $d$ less than 4 ), the automatizability question for $A C_{d}^{0}$ is still unresolved. Using different techniques, [1] proved that Resolution is not automatizable unless the fixed parameter hierarchy collapses; however this method does not rule out slightly superpolynomial algorithms.

We denote $\bigwedge_{i} F_{i}$ to be the conjunction of all $F_{i}$ and $\bigvee_{i} F_{i}$ to be the disjunction of all $F_{i}$. As usual $\Sigma_{k}$ and $\Pi_{k}$ are the collection of depth $k$ formulas where the top level gate is an OR and an AND respectively. We also use $\Delta_{k}$ for the set of formulas in both $\Sigma_{k}$ and $\Pi_{k}$.

Definition 10 Let $G$ be a $n$ node graph with constant out-degree 2 where each node is labeled with an integer $x_{i}, x_{i}$ not all zero. The formula $\operatorname{IGOP}(G)$ intuitively expresses that there exists some $i$ with a nonzero label $x_{i}$ at least as large as that of its children. Formally, if $\mathbf{x}(i)$ is a set of variables representing the value $x_{i}$ in binary, $\operatorname{IGOP}(\mathrm{G})$ is the formula

$$
\bigvee_{i} \mathbf{x}(i) \neq 0 \longrightarrow \bigvee_{i}[\mathbf{x}(i)>0] \wedge\left[\mathbf{x}(i) \geq \mathbf{x}\left(c_{1}(i)\right)\right] \wedge\left[\mathbf{x}(i) \geq \mathbf{x}\left(c_{2}(i)\right)\right]
$$

where $c_{1}(i)$ and $c_{2}(i)$ are the children of $i$ and $\geq$ denotes a standard $\Delta_{2}^{+}$formula for comparison. 
Note that $\left[\mathbf{x}(i) \geq \mathbf{x}\left(c_{1}(i)\right)\right] \wedge\left[\mathbf{x}(i) \geq \mathbf{x}\left(c_{2}(i)\right)\right]$ is $N C^{0}$ of $\Delta_{2}^{+}$which can be written in $\Delta_{2}^{+}$so that $\operatorname{IGOP}(G)$ is in $\Sigma_{2}^{+}$formula.

Definition 11 The formula Max-Node $n$ intuitively expresses that in any set of $n$ integers, there exists a maximal element. Formally, let $\mathbf{x}(i)$ be the set of variables represending the value $x_{i}$

$$
\operatorname{Max}^{-N_{o d e}}=\bigvee_{i} \bigwedge_{j \neq i}[\mathbf{x}(i) \geq \mathbf{x}(j)]
$$

For our purposes, we'd like to apply the IGOP principle to graphs where the nodes are labeled with differences of integers. This does not inherently add to the depth $\operatorname{IGOP}(G)$ since we will develop a $\Delta_{2}^{+}$circuit to compare difference (see Sect. 4).

Definition 12 Let $\Sigma_{2}^{+}$-Frege+IGOP denote the $\Sigma_{2}^{+}$-Frege proof system augmented with the following axiom schema for IGOP. Let $\mathbf{d}(i)$ be shorthand for the difference $|\mathbf{x}(i)-\mathbf{y}(i)|$

$$
\left.\bigvee_{i}[\mathbf{x}(i) \neq \mathbf{y}(i)] \longrightarrow \bigvee_{i}[\mathbf{d}(i)>0] \wedge[\mathbf{d}(i)) \geq \mathbf{d}\left(c_{1}(i)\right)\right] \wedge\left[\mathbf{d}(i) \geq \mathbf{d}\left(c_{2}(i)\right)\right]
$$

which states intuitively that if $\mathbf{x}$ and $\mathbf{y}$ are not equal for every $i$ then there exists an $i$ where the difference $\mathbf{d}(i)$ is nonzero at least as the value of $\mathbf{d}$ at the children of $i$.

We will show that $I G O P$ has a $\Sigma_{3}$ proof (via Max-Node ${ }_{n}$ ), $\Sigma_{2}^{+}$-Frege+IGOP is a special case of $\Sigma_{3}$-Frege.

\section{Main Result}

In this section we prove our main theorem, showing that if $\Sigma_{3}$-Frege admits feasible interpolation, then SSG can be solved in polynomial time.

Theorem 13 If $\Sigma_{2}^{+}$-Frege+IGOP has feasible interpolation, then SSG is in P.

Corollary 14 If $\Sigma_{3}$-Frege has feasible interpolation, then SSG is in P.

The main idea behind the reduction is as follows. Given an SSG, $G$, we first construct an $m$-stopping game $G^{\prime}$, where $G^{\prime}$ has value greater than $1 / 2$ if and only if $G$ has value greater than $1 / 2$. This implies that the following statement is unsatisfiable.

$$
F_{G^{\prime}}=\left(I_{G^{\prime}}(\vec{x})=\vec{x} \wedge \vec{x}(0)>\frac{1}{2}\right) \bigwedge\left(I_{G^{\prime}}(\vec{w})=\vec{w} \wedge \vec{w}(0) \leq \frac{1}{2}\right),
$$

where each $\vec{x}(i)$ and $\vec{w}(i)$ is an integer represented by a length $N$ binary string where $N$ is $O(n)$.

For every stopping game $G^{\prime}$ we will prove that $F_{G^{\prime}}$ has a polynomial-sized $\Sigma_{3}$-Frege refutation. Thus if $\Sigma_{3}$-Frege has feasible interpolation, then the interpolant for $F_{G}^{\prime}$ solves the SSG-value problem for $G$. In order to provide a short $\Sigma_{3}$-Frege refutation of $F_{G}^{\prime}$, it suffices to provide a polynomial-sized $\Sigma_{3}$-Frege proof of

$$
\operatorname{Uniqueness}(G)=" I_{G}(\vec{x})=\vec{x}, I_{G}(\vec{w})=\vec{w} \longrightarrow \vec{x}=\vec{w} "
$$

To see this, note that if Uniqueness $(G)$ has a short $\Sigma_{3}$-Frege proof, then: (1) From $I_{G}(\vec{x})=\vec{x}$ and $I_{G}(\vec{w})=\vec{w}$, we can derive $\vec{x}=\vec{w}$; (2) Secondly, from $\vec{x}=\vec{w}$, we can derive $\vec{x}(0)=\vec{w}(0)$; and finally (3) From $\vec{x}(0)=\vec{w}(0)$, we can derive $\vec{x}(0) \leq \frac{1}{2}$, which contradicts $F_{G}$. 
Theorem 15 For any stopping simple stochastic game $G$, the statement Uniqueness $(G)$ can be proved in $\Sigma_{2}^{+}$-Frege $+I G O P$.

We first present a proof of Theorem 6, the uniqueness theorem for SSGs. The remainder of our paper will then focus on showing that this can be formalized in depth-3 Frege. The proof of Theorem 15 will use the arithmetic formulas and lemmas developed in Sect. 4 to translate the proof below into a polynomial depth-3 Frege proof with the same structure.

\section{Proof: Theorem 6}

Let $G$ be a $m$-stopping game and suppose that $\vec{w}$ and $\vec{x}$ are both stable for $G$ and $\vec{x} \neq \vec{w}$. Denote $\vec{d}$ the difference vector, $\vec{d}=|\vec{w}-\vec{x}|$. Let $i$ be a node such that $\vec{d}(i)>0, \vec{d}(i) \geq \vec{d}(j)$ and $\vec{d}(i) \geq \vec{d}(k)$ where $j$ and $k$ are the original children of $i$. Note that such an $i$ exists since choosing $i$ that maximizes $\vec{d}(i)$ satisfies these conditions. We will show that the structure of the stopping game forces one of $\vec{d}(j)>\vec{d}(i)$ or $\vec{d}(k)>\vec{d}(i)$.

It is useful to recall that $\vec{w}\left(e_{s, l}\right)$ for every new average node in $G^{\prime}$, is exactly determined by $\vec{w}(s)$ by the construction of the stopping game. $\vec{w}\left(e_{s, l}\right)=\left(1-\frac{1}{2^{s}}\right) \vec{w}(l)$, and in particular $\vec{w}\left(e_{m, l}\right)=$ $\left(1-\frac{1}{2^{m}}\right) \vec{w}(l)$. And likewise for $\vec{x}$.

Suppose without loss of generality that $\vec{x}(i)>\vec{w}(i)$, so that $\vec{d}(i)=\vec{x}(i)-\vec{w}(i)$.

Case 1. $i$ is a max or min node. We will show the proof when $i$ is a max node; the same argument follows for min nodes by symmetry. Suppose that both $\vec{w}(j) \geq \vec{w}(k)$ and $\vec{x}(j) \geq \vec{x}(k)$. Then

$$
\begin{aligned}
\vec{d}(i) & =\vec{x}(i)-\vec{w}(i)=\max \left(\vec{x}\left(e_{m j}\right), \vec{x}\left(e_{m k}\right)\right)-\max \left(\vec{w}\left(e_{m j}\right), \vec{w}\left(e_{m k}\right)\right) \\
& =\left(1-\frac{1}{2^{m}}\right)(\max (\vec{x}(j), \vec{x}(k))-\max (\vec{w}(j), \vec{w}(k))) \\
& \leq\left(1-\frac{1}{2^{m}}\right)(\vec{x}(j)-\vec{w}(j)) \leq\left(1-\frac{1}{2^{m}}\right) \vec{d}(j)<\vec{d}(j)
\end{aligned}
$$

If instead $\vec{w}(k) \geq \vec{w}(j)$ and $\vec{x}(k) \geq \vec{x}(j)$ the same proof holds. On the other hand, if $\vec{w}(k) \geq$ $\vec{w}(j)$, then repeating the above we prove that $\vec{d}(i)<\vec{x}(j)-\vec{w}(k)$. But since $\vec{w}(k) \geq \vec{w}(j)$ (and all values are positive), $\vec{d}(j)=\vec{x}(j)-\vec{w}(j) \geq \vec{x}(j)-\vec{w}(k)>\vec{d}(i)$.

Case 2. $i$ is an average node. Then

$$
\begin{aligned}
\vec{d}(i) & =\vec{x}(i)-\vec{w}(i)=\frac{1}{2} \vec{x}\left(e_{m j}\right)+\frac{1}{2} \vec{x}\left(e_{m k}\right)-\frac{1}{2} \vec{w}\left(e_{m j}\right)-\frac{1}{2} \vec{w}\left(e_{m k}\right) \\
& =\frac{1}{2} \cdot\left(1-\frac{1}{2^{m}}\right)[(\vec{x}(j)-\vec{w}(j))+(\vec{x}(k)-\vec{w}(k))] \\
& \leq\left(1-\frac{1}{2^{m}}\right)\left[\frac{1}{2} \vec{d}(j)+\frac{1}{2} \vec{d}(k)\right]<\frac{1}{2} \vec{d}(j)+\frac{1}{2} \vec{d}(k)
\end{aligned}
$$

$\frac{1}{2} \vec{d}(j)+\frac{1}{2} \vec{d}(k)>\vec{d}(i)$ implies that at least one of $\vec{d}(j)>\vec{d}(i)$ or $\vec{d}(k)>\vec{d}(i)$ 


\section{Arithmetic Formulas}

We will represent integers using two's complement binary notation. In two's complement a set of variables $z_{1} z_{2} \ldots z_{n}$ has the value $z=-2^{n-1} \cdot z_{1}+2^{n-2} \cdot z_{2}+\ldots+2^{1} \cdot z_{n-1}+2^{0} \cdot z_{n}$. Positive numbers will always have a 0 in the first bit and can be read as a standard unsigned binary integer. The advantage of 2's complement will be clear when subtraction is defined. Subtraction can simply be treated as adding negative numbers and all sums that do not result in an overflow will automatically have the correct signed value.

To prevent overflow, we will also pad each integer by a constant $k$ where $k$ is the largest number of summands used in any operation so that $z_{1} z_{2} \ldots z_{n} \rightarrow z_{1}^{k} z_{2} z_{3} \ldots z_{n}$. For positive numbers this means $\mathrm{k} 0$ 's at the beginning and for negative numbers this means $\mathrm{k} 1 \mathrm{~s}$.

Let $\vec{x}$ be a vector that satisfies $\vec{x}=I_{G}(\vec{x})$ for some game G. Recall that in any SSG, the values can be written as $\frac{a}{b}$ where $b=O\left(2^{n}\right)$. After normalizing to a common denominator $D$ and padding by $k$, each $x(i)$ will be expressed as a set of $N$ binary variables $x_{1} x_{2} \ldots x_{N}$ so that

$$
x(i)=\frac{1}{D}\left(-2^{N-1} \cdot x_{1}+2^{N-2} \cdot x_{2}+\ldots+2^{1} \cdot x_{N-1}+2^{0}\right)
$$

\subsection{Equality}

If $x$ and $y$ are a set of variables $x_{1} x_{2} \ldots x_{N}$ and $y_{1} y_{2} . . y_{N}$ we denote $[x \leftrightarrow y]$ to be the formula $\forall i\left(x_{i} \leftrightarrow y_{i}\right)$. This means that if a set of formulas has depth $\mathrm{k}$ we can also express equality between the two sets in depth $\mathrm{k}$.

\subsection{Addition}

It is well known that addition of two integers can be efficiently computed by depth- 2 circuits. However, most of our proof will require sums of more than two integers. Let $\mathbf{x}$ be an array of integers $k$ integers

$$
\mathbf{x}=\left(\begin{array}{cccc}
x_{11} & x_{12} & \ldots & x_{1 N} \\
x_{21} & x_{22} & \ldots & x_{2 N} \\
\vdots & \vdots & \ddots & \vdots \\
x_{k 1} & x_{k 2} & \ldots & x_{k N}
\end{array}\right)
$$

Denote $\mathbf{x}(i)$ the $i^{\text {th }}$ row of $\mathbf{x}$. Recall that the $i^{\text {th }}$ bit of the sum $\mathbf{x}(1)+\ldots+\mathbf{x}(k)$ can be computed as the parity of $x_{j i}$ for all $j$ and the carry bits entering column $i$. The main difficulty is calculating these carry bits in low depth.

We will use a carry circuit developed by Atserias and Maneva [4]. The basic idea is similar to addition of two integers in that we want to express each carry bit $c_{i}$ in a formula that does not depend linearly on previous carry bits $c_{j}, j>i$. [4] shows that computing carries can by done in $\Delta_{2}$ for any constant $k$ number of summands.

For any constant $k$ there exists a state machine with $k+1$ states computing the number of carry bits generated by the addition of $k$ integers. The circuit for $k=4$ is pictured below.

Denote $\mathbf{x}_{i}=\sum_{j=1}^{k} \mathbf{x}_{j i}$ be the bitwise sum for the $i^{\text {th }}$ column of $\mathbf{x}$. Then the state machine in Fig. 3 computes whether the sum of $\mathbf{x}$ has at least one bit of overflow when given input $\mathbf{x}_{1} \mathbf{x}_{2} \ldots \mathbf{x}_{N}$. 


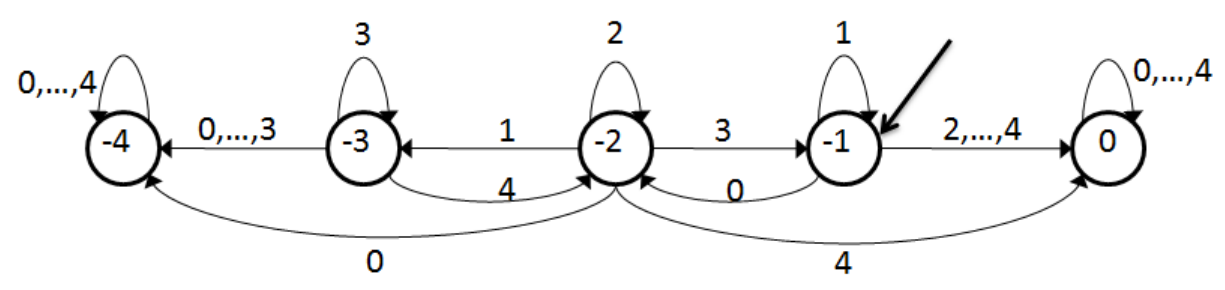

Figure 3: Carry state machine for the sum of four integers. The start state is the state labelled by " 1 " and the accept state is labelled " 0 ". The machine accepts iff at least one overflow is generated by the sum

Each state can be interpreted as a range of possible values for the partial sum $\sum_{i=1}^{p} 2^{p-i} \cdot \mathbf{x}_{i}$ after reading the first $p$ values. The accept sink represents the range $\left[2^{p}, k \cdot 2^{p}\right]$, the reject sink represents the range $\left[0,2^{p}-k\right]$ and each intermediate state represents the exact number $2^{p}-s$ where $-s$ is the label of the state. In other words, the intermediate states are reached only if the partial sum is "close to" $2^{p}-1$. All transitions are chosen to maintain this invariant.

$$
\text { State }-s \text { and input } \mathbf{x}_{i} \text { has a transition to } \begin{cases}-2 s+\mathbf{x}_{i} & \text { if }-2 s+\mathbf{x}_{i} \in[-k, 0] \\ 0 & \text { if }-2 s+\mathbf{x}_{i}>0 \\ -k & \text { if }-2 s+\mathbf{x}_{i}<-k\end{cases}
$$

Lemma 16 The above described circuit correctly computes the overflow bit for addition of $k$ integers.

Proof:(Based on Atserias \& Maneva '10)

Each intermediate state reached correctly represents the value of the partial sum. Suppose that the state at step $p-1$ is $-s$ where $-s$ is not a sink and $s=\sum_{i=1}^{p-1} 2^{p-1-i} \mathbf{x}_{i}-2^{p-1}$. Then after the transition at $p$ the new state $s^{\prime}$ is determined by $-2 s+\mathbf{x}_{p}=2 \cdot \sum_{i=1}^{p-1} 2^{p-1-i} \mathbf{x}_{i}-2^{p}+\mathbf{x}_{p}=$ $\sum_{i=1}^{p} 2^{p-i} \mathbf{x}_{i}-2^{p}$. As long as this value is in the range $[-k, 0], s^{\prime}$ is correct.

If at any point we reach the accept sink, there is some $p$ where $\sum_{i=1}^{p} \mathbf{x}_{i} \geq 2^{p}$. Then the entire sum $\sum_{i=1}^{N} \mathbf{x}_{i} \geq 2^{p} \cdot 2^{N-p}+0 \geq 2^{N}$ and there is an overflow. If instead we reach the the "-k" state, then there is a $p$ where $\sum_{i=1}^{p} \mathbf{x}_{i} \leq 2^{p}-k$ in which case $\sum_{i=1}^{N} \mathbf{x}_{i} \leq\left(2^{p}-k\right) \cdot 2^{N-p}+k \cdot\left(2^{N-p}-1\right) \leq 2^{N}-1$ and there is no overflow. Thus "-k" can also be thought of as a reject state.

Observation 17 At any point during the computation if we have not yet reached a terminal state it is sufficient to look at the previous $\ell$ inputs to determine the current intermediate state where $\ell$ is any number $>\lfloor\log k\rfloor$.

Suppose that we have not yet reached a terminal state at bit $p$. Then the possibilities for $\sum_{i=1}^{p} \mathbf{x}_{i}$ are really quite limited, as only the range $\left[2^{p}-k+1,2^{p}-1\right]$ is possible. To distinguish between them its sufficient to know $\sum_{i=1}^{p} \mathbf{x}_{i}$ MOD $k$ which is entirely determined by the $\ell$ trailing bits. 
Let $S(i, \mathbf{x})$ represent the state of the computation at $i$ if a sink has not yet been reached

$$
S(i, \mathbf{x})= \begin{cases}\left(2^{\ell} \mathbf{x}_{i-\ell}+\ldots+2^{0} \mathbf{x}_{i} \operatorname{MOD} \ell\right)-2^{\ell} & \text { if } i \geq \ell, \\ \left(2^{i} \mathbf{x}_{1}+\ldots+2^{0} \mathbf{x}_{i} \operatorname{MOD} i\right)-2^{i} & \text { if } i<\ell,\end{cases}
$$

The important thing to note is that $S(i, \mathbf{x})$ only depends on a constant number of bits. Observation 17 implies that we can define constant size formulas $A(i)$ and $R(i)$ where $A(i)$ represents whether or not the transition taken from $i$ leads to an accept, and $R(i)$ represents whether or not the transition taken from $i$ leads to reject.

$$
\begin{gathered}
{[A(i, \mathbf{x})] \text { is true if and only if } 2 \cdot S(i-1, \mathbf{x})+\mathbf{x}_{i} \geq 0} \\
{[R(i, \mathbf{x})] \text { is true if and only if } 2 \cdot S(i-1, \mathbf{x})+\mathbf{x}_{i} \leq-k}
\end{gathered}
$$

As long as a terminal state has not yet been reached at some $j<i$, then $[A(i, \mathbf{x})]$ is correct. In fact we really only care if the reject sink has not been reached so we can define the formula

$$
\left[C^{1}(\mathbf{x})\right] \stackrel{\text { def }}{=} \bigvee_{j \geq 1} \neg[R(1, \mathbf{x})] \wedge \ldots \wedge \neg[R(j-1, \mathbf{x})] \wedge[A(j, \mathbf{x})]
$$

At least one bit of overflow is generated in the sum of $\mathbf{x}$ if and only if the above formula is true. Furthermore, overflow is not generated if and only if there is some bit $j$ at which the circuit reaches reject and every bit from $j$ to $i$ does not prematurely reach accept.

$$
\neg\left[C^{1}(\mathbf{x})\right]=\bigvee_{j \geq 1} \neg[A(1, \mathbf{x})] \wedge \ldots \wedge \neg[A(j-1, \mathbf{x})] \wedge[R(j, \mathbf{x})]
$$

By negating the above formula, we have an equivalent definition of $\left[C^{1}(\mathbf{x})\right]$ with a top level AND so $\left[C^{1}(\mathbf{x})\right]$ is in $\Delta_{2}^{+}$.

We now have a low depth formula calculating whether overflow occurs in the sum of $\mathbf{x}$, ie whether there is at least one carry leaving the most significant bit of the sum. However, our greater goal is to compute the sum of two numbers so we need to know not just that a carry is generated, but also exactly how many carry bits are generated. For that we only need to modify the beginning bits of $\mathbf{x}$. Let $s$ be the $\ell$-bit binary representation of $2^{\ell}-d$ then at least $d$ bits of carry are generated in the sum of $\mathbf{x}$, if and only if at least one bit of overflow is generated in the sum of $\mathbf{x}^{\prime}$ where

$$
\mathbf{x}^{\prime}=\left(\begin{array}{ccccccc}
s_{1} & s_{2} & \ldots & s_{\ell} & x_{11} & \ldots & x_{1 N} \\
0 & 0 & \ldots & 0 & x_{21} & \ldots & x_{2 N} \\
\vdots & \vdots & \ddots & \vdots & \vdots & \ddots & \vdots \\
0 & 0 & \ldots & 0 & x_{k 1} & \ldots & x_{k N}
\end{array}\right)
$$

This formulation is equivalent to starting the carry circuit in state $-d$; the partial sum of $\mathbf{x}^{\prime}$ at bit $\ell$ is exactly $2^{\ell}-d$.

In addition to the exact number of carries generated at the most significant bit we also need to know the exact number of carries generated at each intermediate bit. Since there was nothing special about the starting bit, we need only run the carry circuit starting from bit $j$ rather than bit 0 to find the number of carries entering $j-1$. Putting this all together, the following (constant size) formulas can be used to calculate whether $d$ bits of carry enter bit $i$. 


$$
S^{d}(\mathbf{x}, i, j)=\left\{\begin{array}{c}
\left(2^{\ell} x_{j-\ell}+\ldots+2^{0} x_{j} \operatorname{MOD} \ell\right)-2^{\ell} \\
\quad \text { if } j-i \geq \ell \\
\left(2^{\ell} s_{\ell-(j-i)-1}+\ldots+2^{(j-i)+1} s_{\ell}+2^{(j-i)} x_{i}+\ldots+2^{0} x_{j} \operatorname{MOD} i\right)-2^{\ell} \\
\quad \text { if } j-i<\ell
\end{array}\right.
$$

Intuitively, $S^{d}(\mathbf{x}, i, j)$ is equivalent to $S\left(j, \mathbf{x}^{\prime}\right)$, ie the state of the circuit at bit $j$ in the sum of $\mathbf{x}^{\prime}$ where $s=2^{\ell}-d$ and $\mathbf{x}^{\prime}$ is the modified array

$$
\mathbf{x}^{\prime}=\left(\begin{array}{ccccccc}
s_{1} & s_{2} & \ldots & s_{\ell} & x_{1 i} & \ldots & x_{1 N} \\
0 & 0 & \ldots & 0 & x_{2 i} & \ldots & x_{2 N} \\
\vdots & \vdots & \ddots & \vdots & \vdots & \ddots & \vdots \\
0 & 0 & \ldots & 0 & x_{k i} & \ldots & x_{k N}
\end{array}\right)
$$

As before, $S($ ) depends on a constant number of bits so we can define constant sized formulas such that

$$
\begin{gathered}
{\left[A^{d}(\mathbf{x}, i, j)\right] \text { is true if and only if } 2 \cdot S^{d}(\mathbf{x}, i, j-1)+\mathbf{x}_{j} \geq 0} \\
{\left[R^{d}(\mathbf{x}, i, j)\right] \text { is true if and only if } 2 \cdot S^{d}(\mathbf{x}, i, j-1)+\mathbf{x}_{j} \leq-k} \\
{\left[C^{d}(\mathbf{x}, i)\right] \stackrel{\text { def }}{=} \bigvee_{j>i} \neg\left[R^{d}(\mathbf{x}, i, i+1)\right] \wedge \ldots \wedge \neg\left[R^{d}(\mathbf{x}, i, j-1)\right] \wedge\left[A^{d}(\mathbf{x}, i, j)\right]}
\end{gathered}
$$

$\left[C^{d}(\mathbf{x}, i)\right]$ is true iff at least $d$ bits of carry enter bit $i$ in the sum of $\mathbf{x}$. Finally, to write $\left[C^{d}()\right]$ with a top level $\bigwedge$ gate we use the same approach as for the summation of two integers.

$$
\neg\left[C^{d}(\mathbf{x}, i)\right] \stackrel{\text { def }}{=} \bigvee_{j>i} \neg\left[A^{d}(\mathbf{x}, i, i+1)\right] \wedge \ldots \wedge \neg\left[A^{d}(\mathbf{x}, i, j-1)\right] \wedge\left[R^{d}(\mathbf{x}, i, j)\right]
$$

For any $i$ and $d, C^{d}(\mathbf{x}, i)$ is in $\Delta_{2}^{+}$. Then the summation of any set of integers $S U M(\mathbf{x})$ can be calculated as

$$
S U M(\mathbf{x})_{i} \stackrel{\text { def }}{=} \operatorname{MOD}_{2}\left(x_{1 i}, x_{2 i}, \ldots, x_{k i},\left[C^{1}(\mathbf{x}, i)\right], \ldots,\left[C^{k}(\mathbf{x}, i)\right]\right)
$$

The $i^{\text {th }}$ bit of the sum is the parity of the $i^{\text {th }}$ bit of each input and all carry bits entering at $i$. When $k$ is constant, the formula above is $N C_{0}$ of $\Delta_{2}^{+}$which is in $\Delta_{2}^{+}$as desired. When convenient, we will also use $[\mathbf{x}(1)+\ldots+\mathbf{x}(k)]$ interchangeably with $S U M(\mathbf{x})$.

\subsection{Subtraction}

Using two's complement numbers we can express a difference $y-x$ as $y+(-x)$. Let $x$ be the two's complement expression of $\mathrm{x}$. Then regardless of the sign of $\mathrm{x}$, the two's complement expression of $-\mathrm{x}$ is $\bar{x}+1$ where $\bar{x}$ denotes the bitwise complement of $x$. 
First we show that $-\mathrm{x}=\bar{x}+1$ whenever $\mathrm{x}$ is non-negative. $\mathrm{x}$ non negative implies that $x_{1}=0$ and $x_{[2: N]}$ is exactly the unsigned binary representation of $\mathrm{x}$.

$$
\begin{aligned}
\mathrm{x} & =-2^{N-1} x_{1}+2^{N-2} x_{2}+\ldots+x_{N} \\
& =2^{N-2} x_{2}+\ldots+x_{N} \\
\left(2^{N-1}-1\right)-\mathrm{x} & =2^{N-2} \bar{x}_{2}+\ldots+\bar{x}_{N} \\
-1-\mathrm{x} & =2^{N-1} \bar{x}_{1}+2^{N-2} \bar{x}_{2}+\ldots+\bar{x}_{N}
\end{aligned}
$$

And $\bar{x}+1$ is exactly the two's complement representation of $-\mathrm{x}$.

Similarly when $\mathrm{x}$ is negative,

$$
\begin{aligned}
\mathrm{x} & =-2^{N-1} x_{1}+2^{N-2} x_{2}+\ldots+x_{N} \\
& =-2^{N-1}+2^{N-2} x_{2}+\ldots+x_{N} \\
2^{N-1}+\mathrm{x} & =2^{N-2} x_{2}+\ldots+x_{N} \\
\left(2^{N-1}-1\right)-2^{N-1}-\mathrm{x} & =2^{N-2} \bar{x}_{2}+\ldots+\bar{x}_{N} \\
-1-\mathrm{x} & =2^{N-1} \bar{x}_{1}+2^{N-2} \bar{x}_{2}+\ldots+\bar{x}_{N}
\end{aligned}
$$

The same trick of course works for any constant number of integers. Define subtraction as

$$
[\mathbf{x}(1)+\ldots+\mathbf{x}(k)-\mathbf{y}(1)-\ldots-\mathbf{y}(l)] \stackrel{\text { def }}{=}[\mathbf{x}(1)+\ldots+\mathbf{x}(k)+\overline{\mathbf{y}(1)}+1+\ldots+\overline{\mathbf{y}(l)}+1]
$$

\subsection{Comparisons}

To compare to numbers we need only look at the sign bit of their difference.

$$
[x \geq y] \stackrel{\text { def }}{=} \neg[x+\bar{y}+1]_{1}
$$

Strictly greater than will always be a negation of greater than, $[y<x] \stackrel{\text { def }}{=} \neg[x \geq y]$. In general, any number of integers can be compared using the same method.

$$
\left[x_{1}+\ldots+x_{k}-y_{1}-\ldots-y_{l} \geq 0\right] \stackrel{\text { def }}{=} \neg\left[x_{1}+\ldots+x_{k}+\overline{y_{1}}+1+\ldots+\overline{y_{l}}+1\right]_{1}
$$

\subsection{Max and Min}

Let $[\max (x, y)]_{i}$ be the following formula representing the $i^{\text {th }}$ bit of $\max (x, y)$ then $[\max (x, y)]_{i}$ is true if and only if

$$
[x \geq y] \wedge x_{i} \bigvee \neg[x \geq y] \wedge y_{i} .
$$

Similarly, we have $\left.[\min (x, y)]_{i}\right]$ is true if and only if

$$
\neg[x \geq y] \wedge x_{i} \bigvee[x \geq y] \wedge y_{i}
$$

As written above max and min are $\Sigma_{3}^{+}$formulas. To simplify this to a $\Sigma_{2}^{+}$formula write $[x \geq y]$ in $\Sigma_{2}^{+}$and then factor in the variables $x_{i}$ (or $y_{i}$ ). As such, max and min become formulas that look like 


$$
\left(x_{i} \wedge f_{1}\right) \vee\left(x_{i} \wedge f_{2}\right) \vee \ldots \vee\left(x_{i} \wedge f_{n}\right) \vee\left(y_{i} \wedge g_{1}\right) \vee\left(y_{i} \wedge g_{2}\right) \vee \ldots \vee\left(y_{i} \wedge g_{n}\right)
$$

where each $f_{i}$ and $g_{i}$ is a conjunction of constant size formulas (ANDs of small ORs). The same technique can be used to reduce the above formula to $\Pi_{2}^{+}$placing $\max$ and $\min$ in $\Delta_{2}^{+}$as claimed.

\subsection{Bit-shifting}

In order to express $x=I_{G}(x)$ we also need to express the quantity $\left(1-\frac{1}{2^{m}}\right) x(i)$.

Let $r \operatorname{Shift}(x, m)$ denote the bit string $x$ shifted right by $m$ bits so that $x_{1}, . ., x_{N}$ becomes $0^{m}, x_{1}, \ldots, x_{N-m-1}, x_{N-m}$. Then $\frac{1}{2^{m}} x(i)$ is exactly the number represented by $\operatorname{rShift}(x, m)$ and $\left(1-\frac{1}{2^{m}}\right) x(i)$ can be expressed as $[x-\operatorname{rShift}(x, m)]$

In general performing bit-shifting can result in a loss of information if less significant bits get "shifted off" to the right. However, as long as we apply this only to values of the SSG this cannot occur since $\left(1-\frac{1}{2}^{m}\right) x(i)$ is a value in $\mathrm{G}$ for every node $i$ and all values in $\mathrm{G}$ are known to have bounded $N$-bit precision.

Similarly, let $\operatorname{lShift}(x, m)$ denote the bit string $x$ shifted by $m$ bits to the left $x_{1} \ldots x_{N} \rightarrow$ $x_{m}+1 \ldots x_{N} 0^{m}$. Just as $r$ Shift() corresponds to division by 2 , lShift() corresponds to multiplication by two. Again, we need to be careful not to lose any bits. As we have already padded all inputs as long as the shift amount is less than the padding this operation is sound. In the actual proof, lShift $(x, m)$ is only used for $m=1$

\subsection{Summary of Notation}

For the remainder of the paper $[F(x)]$ will be used to distinguish the logical formula version of $F(x)$ from the mathematical formula. To simpify notation, only one set of brackets will be used when any nesting occurs so that $[[F(x)] \leftrightarrow[G(x)]]$ is just $[F(x) \leftrightarrow G(x)]$.

Boldface variables $\mathbf{x}$ denote an array of $k \times N$ array of integers and $\mathbf{x}(i)$ is the $i^{\text {th }}$ row. Normal font variables $x$ represent a single integer expressed with $N$ binary variables.

- $[x \leftrightarrow y]$ is a formula that is true if and only if $\forall i\left(x_{i} \leftrightarrow y_{i}\right)$

- $[\mathbf{x}(1)+\ldots+\mathbf{x}(k)-\mathbf{y}(1) \ldots-\mathbf{y}(l)]$ represents a set of $N$ formulas, such that the $i^{\text {th }}$ formula is true iff the $i^{\text {th }}$ bit of $\mathbf{x}(1)+\ldots+\mathbf{x}(k)-\mathbf{y}(1) \ldots-\mathbf{y}(k)$ is 1 .

- $[\mathbf{x}(1)+\ldots+\mathbf{x}(k)-\mathbf{y}(1) \ldots-\mathbf{y}(l)] \geq z]$ is true if and only if the sum on the left is $\geq z$. And similarly $[\mathbf{x}(1)+\ldots+\mathbf{x}(k)-\mathbf{y}(1) \ldots-\mathbf{y}(l)]<z]$ is true iff the sum is less than $z$

- $[\max (x, y)]$ represents a set of formulas where the $i^{\text {th }}$ formula is $x_{i}$ iff $x=\max (x, y)$ and $[\min (x, y)]$ represents the set of formulas where the $i^{\text {th }}$ formula is $x_{i}$ iff $x=\min (x, y)$.

\subsection{Arithmetic Lemmas}

The following lemmas comprise the bulk of the work of our proof. (Proofs are in Appendix A.)

Lemma 18 (Substitution) For any $\Delta_{2}^{+}$formula $F\left(x_{1}, x_{2}, \ldots x_{N}, z \ldots\right)$ there exists a short PK proof of the following sequent, where all formulas in the proof are $\Delta_{2}^{+}$formulas.

$$
[x \leftrightarrow y] \longrightarrow\left[F\left(x_{1}, x_{2}, \ldots x_{N}, z \ldots\right) \leftrightarrow F\left(y_{1}, y_{2}, \ldots y_{N}, z \ldots\right)\right]
$$


Lemma 19 For $N$ bit integers $x$ and $y$ the following sequents have short $P K$ proofs where all formulas are $\Delta_{2}^{+}:(1)[x \geq y] \longrightarrow[\max (x, y)=x]$, and (2) $[x \geq y] \longrightarrow[\min (x, y)=y]$.

Lemma 20 (Nested Addition) For any $N$ bit integers $a, b, c$, and any array of constant $k$ $N$-bit integers $\mathbf{x}$ there exists short $P K$ proof of the following where all formulas are $\Delta_{2}^{+}$:

$$
\begin{array}{r}
{[a+b \leftrightarrow c] \longrightarrow[\mathbf{x}(1)+\ldots+\mathbf{x}(k)+a+b \leftrightarrow \mathbf{x}(1)+\ldots+\mathbf{x}(k)+c]} \\
{[a-b \leftrightarrow c] \longrightarrow[\mathbf{x}(1)+\ldots+\mathbf{x}(k)+a+\bar{b}+1 \leftrightarrow \mathbf{x}(1)+\ldots+\mathbf{x}(k)+c]}
\end{array}
$$

Corollary 21 Given $\left[x+a+b \leftrightarrow 1^{N}\right]$ we can derive $[\bar{x} \leftrightarrow a+b]$ for any integers $x$, $a$, $b$. In particular, $[x \leftrightarrow a-b] \longrightarrow[\bar{x} \leftrightarrow \bar{a}+b]$, and $[x \leftrightarrow a+b] \longrightarrow[\bar{x} \leftrightarrow \bar{a}-\bar{b}]$.

Lemma 22 Let $x, y, x^{\prime}, y^{\prime}$ be $N$-bit integers, and $m>0 . \frac{1}{2^{m}} y$ is the set of variables $0^{m} y_{1} y_{2} \ldots y_{N-m}$ Then there exists a short PK proof of the following formula, where all sequent are $\Delta_{2}^{+}$:

$$
\left[x>x^{\prime}\right],\left[x \leftrightarrow y-\frac{1}{2^{m}} y\right],\left[x^{\prime} \leftrightarrow y^{\prime}-\frac{1}{2^{m}} y^{\prime}\right] \longrightarrow\left[x-x^{\prime}-y+y^{\prime}<0\right]
$$

Lemma 23 Fix an $a \times N$ array $\mathbf{z}$ and $a b \times N$ array $\mathbf{x}$ where each integer is padded by $k=a+b$ bits. There is a short proof of the following:

$$
\begin{aligned}
& {[\mathbf{z}(1)+\ldots \mathbf{z}(a)<0],[\mathbf{x}(1)+\ldots+\mathbf{x}(b)<0] \longrightarrow[\mathbf{z}(1)+\ldots \mathbf{z}(a)+\mathbf{x}(1)+\ldots \mathbf{x}(b)<0]} \\
& {[\mathbf{z}(1)+\ldots \mathbf{z}(a) \geq 0],[\mathbf{x}(1)+\ldots+\mathbf{x}(b) \geq 0] \longrightarrow[\mathbf{z}(1)+\ldots \mathbf{z}(a)+\mathbf{x}(1)+\ldots \mathbf{x}(b) \geq 0]}
\end{aligned}
$$

\section{$5 \quad$ Proof of Uniqueness}

Equipped with the above lemmas, we can now prove uniqueness for SSGs in depth-3 Frege. Let $\mathbf{x}$ and $\mathbf{w}$ be arrays of integers as defined in the previous section such that each integer is padded by $k$ bits and $\mathbf{x}(i)$ is the value associated with the $i^{t h}$ node of a $m$-stopping game $\mathrm{G}$. We define a formula, Uniqueness $(\mathrm{G})$ on the $N \cdot(n+m n)$ variables defined above which will state that if $\mathbf{x}=I_{G}(\mathbf{x})$ and $\mathbf{w}=I_{G}(\mathbf{w})$, then $\mathbf{x}(i)=\mathbf{w}(i)$ for every $i$. The premise $\mathbf{x}=I_{G}(\mathbf{x})$ can be stated as follows, using the arithmetic formulas defined in the previous section.

(1) For every max node $i$, with original children $j$ and $k,[\mathbf{x}(i) \leftrightarrow \max (\mathbf{x}(j), \mathbf{x}(k))]$

(2) For every min node $i$, with original children $j$ and $k,[\mathbf{x}(i) \leftrightarrow \min (\mathbf{x}(j), \mathbf{x}(k))]$

(3) For every average node $i$, with original children $j$ and $k$, $[2 \cdot \mathbf{x}(i) \leftrightarrow \mathbf{x}(j)+\mathbf{x}(k)]$

(4) For every average node added to form the stopping game $e_{i j},\left[\mathbf{x}\left(e_{i j}\right) \leftrightarrow \mathbf{x}(j)-\frac{1}{2^{i}} \mathbf{x}(j)\right]$.

For any $i$, we will prove that if $d(i)$ (the absolute value of $x(i)-w(i)$ ) is nonzero and at least as large as its neighbors, $d(j)$ and $d(k)$, then we can derive a contradiction. Formally, let $M(i)$ be the formula:

$$
\begin{array}{r}
{[\mathbf{x}(i)>\mathbf{w}(i)], \forall s \in\{j, k\}([\mathbf{x}(i)-\mathbf{w}(i)-\mathbf{x}(s)+\mathbf{w}(s) \geq 0]),} \\
\forall s \in\{j, k\}([\mathbf{x}(i)-\mathbf{w}(i)-\mathbf{w}(s)+\mathbf{x}(s) \geq 0])
\end{array}
$$

We will prove for every $i: M(i),\left[\mathbf{x}=I_{G}(\mathbf{x})\right],\left[\mathbf{w}=I_{G}(\mathbf{w})\right] \longrightarrow \perp$.

Finally we can use the IGOP axiom schema to prove that there exists an $i$ such that $M(i)$ holds which allows us to conclude: $\left[\mathbf{x}=I_{G}(\mathbf{x})\right],\left[\mathbf{w}=I_{G}(\mathbf{w})\right] \longrightarrow[\mathbf{x}(1) \leftrightarrow \mathbf{w}(1)], \ldots,[\mathbf{x}(n) \leftrightarrow \mathbf{w}(n)]$. 
Case 1. $i$ is a max node with children $j$ and $k$

As in proof of Theorem 6, we can further divide Case 1 into four cases depending on the sign of $[\mathbf{x}(j)-\mathbf{x}(k)]$ and $[\mathbf{w}(j)-\mathbf{w}(k)]$. Because of the way we defined comparisons, $[a<b]$ is exactly $\neg[a \geq b]$ so it it's not hard to show that exactly one of four sub cases is true.

Case 1a. $\left[\mathbf{x}\left(e_{m j}\right) \geq \mathbf{x}\left(e_{m k}\right)\right],\left[\mathbf{w}\left(e_{m j}\right) \geq \mathbf{w}\left(e_{m k}\right)\right]$

Let $H(\mathbf{x}, \mathbf{w})$ denote the subcase premise $\left[\mathbf{x}\left(e_{m j}\right) \geq \mathbf{x}\left(e_{m k}\right)\right],\left[\mathbf{w}\left(e_{m j}\right) \geq \mathbf{w}\left(e_{m k}\right)\right]$. We prove for contradiction that $M(i), H(\mathbf{x}, \mathbf{w}),\left[\mathbf{x}=I_{G}(\mathbf{x})\right],\left[\mathbf{w}=I_{G}(\mathbf{w})\right] \longrightarrow \perp$

Using Lemma 19 we can prove $\left[\mathbf{x}\left(e_{m j}\right) \geq \mathbf{x}\left(e_{m k}\right)\right] \longrightarrow\left[\max \left(\mathbf{x}\left(e_{m j}\right), \mathbf{x}\left(e_{m k}\right)\right) \leftrightarrow \mathbf{x}\left(e_{m j}\right)\right]$. And by weakening the LHS we have $H(\mathbf{x}, \mathbf{w}) \longrightarrow\left[\max \left(\mathbf{x}\left(e_{m j}\right), \mathbf{x}\left(e_{m k}\right)\right) \leftrightarrow \mathbf{x}\left(e_{m j}\right)\right]$. From the statements forming $\left[\mathbf{x}=I_{G}(\mathbf{x})\right]$ above we can apply $\left[\mathbf{x}(i) \leftrightarrow \max \left(\mathbf{x}\left(e_{m j}\right), \mathbf{x}\left(e_{m k}\right)\right)\right]$ and $\left[\mathbf{x}\left(e_{m j}\right) \leftrightarrow \mathbf{x}(j)-\frac{1}{2^{m}} \mathbf{x}(j)\right]$ to prove

$$
(1):\left[\mathbf{x}=I_{G}(\mathbf{x})\right], H(\mathbf{x}, \mathbf{w}) \longrightarrow\left[\mathbf{x}(i) \leftrightarrow \mathbf{x}(j)-\frac{1}{2^{m}} \mathbf{x}(j)\right]
$$

Similarly, (2) : $\left[\mathbf{w}=I_{G}(\mathbf{w})\right], H(\mathbf{x}, \mathbf{w}) \longrightarrow\left[\mathbf{w}(i) \leftrightarrow \mathbf{w}(j)-\frac{1}{2^{m}} \mathbf{w}(j)\right]$. The next step is to prove the following sequent, which follows from Lemma 22 :

$$
\begin{array}{r}
(3):[\mathbf{x}(i)>\mathbf{w}(i)],\left[\mathbf{x}(i) \leftrightarrow \mathbf{x}(j)-\frac{1}{2^{m}} \mathbf{x}(j)\right],\left[\mathbf{w}(i) \leftrightarrow \mathbf{w}(j)-\frac{1}{2^{m}} \mathbf{w}(j)\right] \longrightarrow \\
{[\mathbf{x}(i)-\mathbf{w}(i)-\mathbf{x}(j)+\mathbf{w}(j)<0]}
\end{array}
$$

Finally to put things together, we apply cut on (1),(2) and (3) to obtain:

$$
(4): M(i), H(\mathbf{x}, \mathbf{w}),\left[\mathbf{x}=I_{G}(\mathbf{x})\right],\left[\mathbf{w}=I_{G}(\mathbf{w})\right] \longrightarrow[\mathbf{x}(i)-\mathbf{w}(i)-\mathbf{x}(j)+\mathbf{w}(j)<0]
$$

which is the same as $M(i), H(\mathbf{x}, \mathbf{w}),\left[\mathbf{x}=I_{G}(\mathbf{x})\right],\left[\mathbf{w}=I_{G}(\mathbf{w})\right] \longrightarrow \perp$.

Case 1b. $\left[\mathbf{x}\left(e_{m j}\right)<\mathbf{x}\left(e_{m k}\right)\right],\left[\mathbf{w}\left(e_{m j}\right)<\mathbf{w}\left(e_{m k}\right)\right]$ is similar to the above.

Case 1c. $\left[\mathbf{x}\left(e_{m j}\right) \geq \mathbf{x}\left(e_{m k}\right)\right],\left[\mathbf{w}\left(e_{m j}\right)<\mathbf{w}\left(e_{m k}\right)\right]$

Here the proof uses the same principles as for case 1a and $1 \mathrm{~b}$ but goes through an additional step. As before we will prove that $\mathbf{x}(i)-\mathbf{w}(i)<\mathbf{x}(j)-\mathbf{w}(j)$ contradicting the hypothesis that $\mathbf{x}(i)-\mathbf{w}(i)>\mathbf{x}(j)-\mathbf{w}(j)$.

Following the pattern above we can prove

$$
(5): M(i), H^{\prime}(\mathbf{x}, \mathbf{w}),\left[\mathbf{x}=I_{G}(\mathbf{x})\right],\left[\mathbf{w}=I_{G}(\mathbf{w})\right] \longrightarrow[\mathbf{x}(i)-\mathbf{w}(i)-\mathbf{x}(j)+\mathbf{w}(k)<0]
$$

Note that rather than $\mathbf{w}(j)$, the above sequent contains $\mathbf{w}(k)$ on the right hand side sum. This is not quite what we want; we need to relate the $\mathbf{x}(i)-\mathbf{w}(i)$ to the difference $\mathbf{x}(j)-\mathbf{w}(j)$. However, what we know is that $\mathbf{w}(j)-\mathbf{w}(k)$ is negative (from the case premise). Thus from Lemma 23 and (5) we can conclude

$$
\begin{aligned}
(6) & : M(i), H^{\prime}(\mathbf{x}, \mathbf{w}),\left[\mathbf{x}=I_{G}(\mathbf{x})\right],\left[\mathbf{w}=I_{G}(\mathbf{w})\right] \longrightarrow \\
& {[\mathbf{x}(i)-\mathbf{w}(i)-\mathbf{x}(j)+\mathbf{w}(k)+\mathbf{w}(j)-\mathbf{w}(k)<0] }
\end{aligned}
$$

Finally, using Lemma 20 to cancel $\mathbf{w}(k)-\mathbf{w}(k)=0$ in the above sum, we arrive at the contradiction desired.

$$
M(i), H^{\prime}(\mathbf{x}, \mathbf{w}),\left[\mathbf{x}=I_{G}(\mathbf{x})\right],\left[\mathbf{w}=I_{G}(\mathbf{w})\right] \longrightarrow[\mathbf{x}(i)-\mathbf{w}(i)-\mathbf{x}(j)+\mathbf{w}(j)<0]
$$


or equivalently

$$
M(i), H^{\prime}(\mathbf{x}, \mathbf{w}),\left[\mathbf{x}=I_{G}(\mathbf{x})\right],\left[\mathbf{w}=I_{G}(\mathbf{w})\right] \longrightarrow \perp
$$

Case 1d. $\left[\mathbf{x}\left(e_{m j}\right)<\mathbf{x}\left(e_{m k}\right)\right],\left[\mathbf{w}\left(e_{m j}\right) \geq \mathbf{w}\left(e_{m k}\right)\right]$ is similar to the above.

Case 2. $i$ is min node is symmetric to Case $1, i$ a max node.

Case 3. $i$ is an average node

For average nodes, it is easy to show that $\mathbf{x}(j)-\mathbf{w}(j)>\mathbf{x}\left(e_{m j}\right)-\mathbf{w}\left(e_{m j}\right)$ and $\mathbf{x}(k)-\mathbf{w}(k)>$ $\mathbf{x}\left(e_{m k}\right)-\mathbf{w}\left(e_{m k}\right)$ just as we did in the max node case using Lemma 22. By Lemma 23 we can combine the two negative sums above to the larger negative sum

$$
\left[\mathbf{x}\left(e_{m j}\right)-\mathbf{w}\left(e_{m j}\right)-\mathbf{x}(j)+\mathbf{w}(j)+\mathbf{x}\left(e_{m k}\right)-\mathbf{w}\left(e_{m k}\right)-\mathbf{x}(k)+\mathbf{w}(k)<0\right]
$$

The goal is to make the substitutions that relate this inequality back to $\mathbf{x}(i)-\mathbf{w}(i)$. We can always add zero to any sum without changing its value, and due to Lemma 20 we can also add a pair of integers that sum to zero. Using this trick we will replace the terms containing $e_{m j}$ and $e_{m k}$ with terms containing $i$.

When $i$ is an average node, $2 \mathbf{x}(i)=\left[\mathbf{x}\left(e_{m j}\right)+\mathbf{x}\left(e_{m k}\right)\right]$. Recall that $2 x(i)$ does not represent a sum but a new set of binary variables $\mathbf{x}(i)_{2} \ldots \mathbf{x}(i)_{N} 0$. This is very convenient as we can now freely use Lemma 20 to add $0=2 \mathbf{x}(i)-2 \mathbf{x}(i)$ and $0=2 \mathbf{w}(i)-2 \mathbf{w}(i)$ to the negative sum above to prove that

$$
\begin{array}{r}
{\left[2 \mathbf{x}(i)-2 \mathbf{x}(i)+2 \mathbf{w}(i)-2 \mathbf{w}(i)+\mathbf{x}\left(e_{m j}\right)-\mathbf{w}\left(e_{m j}\right)-\mathbf{x}(j)+\mathbf{w}(j)\right.} \\
\left.+\mathbf{x}\left(e_{m k}\right)-\mathbf{w}\left(e_{m k}\right)-\mathbf{x}(k)+\mathbf{w}(k)<0\right]
\end{array}
$$

In order to cancel out the $e_{m j}$ and $e_{m k}$ terms, we need to make the following four substitutions:

$$
\begin{array}{ll}
\text { - } 2 \mathbf{x}(i) \rightarrow[\mathbf{x}(i)+\mathbf{x}(i)] & \text { - } 2 \mathbf{w}(i) \rightarrow\left[\mathbf{w}\left(e_{m j}\right)+\mathbf{w}\left(e_{m k}\right)\right] \\
\text { - } \overline{2 \mathbf{x}(i)} \rightarrow\left[\overline{\mathbf{x}\left(e_{m j}\right)}-\overline{\mathbf{x}\left(e_{m k}\right)}\right] & \text { - } \overline{2 \mathbf{w}(i)} \rightarrow[\overline{\mathbf{w}(i)}-\overline{\mathbf{w}(i)}]
\end{array}
$$

After removing the zero terms, we finally arrive at

$$
\left[\mathbf{x}=I_{G}(\mathbf{x})\right],\left[\mathbf{w}=I_{G}(\mathbf{w})\right] \longrightarrow[\mathbf{x}(i)+\mathbf{x}(i)-\mathbf{w}(i)-\mathbf{w}(i)-\mathbf{x}(j)+\mathbf{w}(j)-\mathbf{x}(k)+\mathbf{w}(k)<0]
$$

On the other hand, if $M(i)$ is true then both $[\mathbf{x}(i)-\mathbf{w}(i)-\mathbf{x}(j)+\mathbf{w}(j) \geq 0]$ and $[\mathbf{x}(i)-\mathbf{w}(i)-$ $\mathbf{x}(k)+\mathbf{w}(k) \geq 0]$ are true. Applying Lemma 23 to the two positive sums we can derive that the same sum must be positive.

$$
M(i) \longrightarrow[\mathbf{x}(i)-\mathbf{w}(i)-\mathbf{x}(j)+\mathbf{w}(j)+\mathbf{x}(i)-\mathbf{w}(i)-\mathbf{x}(k)+\mathbf{w}(k) \geq 0]
$$

As with the max/min nodes, we have shown that for all average nodes $i$ from $M(i),[\mathbf{x}=$ $\left.I_{G}(\mathbf{x})\right],\left[\mathbf{w}=I_{G}(\mathbf{w})\right]$ we can derive a contradiction. If we can show that whenever $\mathbf{x}$ and $\mathbf{w}$ do not agree in all bits, $M(i)$ is true for some $i$ then the proof of "Uniqueness $(\mathrm{G})$ " is complete.

We have shown that for any $i, M(i) \longrightarrow$ Uniqueness $(G)$. Using the $I G O P$ axiom schema, we can immediately derive $\bigvee_{i} M(i)$ from which we can conclude $\operatorname{Uniqueness}(G)$. 


\subsection{Proving Max-Node ${ }_{n}$}

To achieve the $\Sigma_{3}$ result, it remains to prove Max-Node ${ }_{n}$. Suppose that $x(i)-w(i)$ is maximal among the first $n-1$ nodes, and $x(n) \geq w(n)(w(n)>x(n)$ follows by symmetry). Either (1) $[x(i)-w(i) \geq x(n)-w(n)]$ or $(2)[x(i)-w(i)<x(n)-w(n)]$. If (1) is true then $x(i)-w(i)$ remains maximal. If $(2)$ is true then for each $j,[x(n)-w(n)-x(j)+w(j)<0]$ would imply that $[x(i)-w(i)-x(j)+w(j)]$ as well (Lemma 23 followed by Lemma 20). Since we know the latter is not true, we can prove that for all $j,[x(n)-w(n) \geq x(j)-w(j)]$. In either case, we've proven Max-Node ${ }_{n}$, by $\Sigma_{3}^{+}$proofs.

\subsection{Removing the bottom fan-in}

So far, we have shown how to prove the uniqueness property with $\Sigma_{3}^{+}$proofs, or with depth $\Sigma_{2}^{+}$ proofs plus the IGOP principle. With one additional idea, we can remove the small bottom fanin. Recall that the bottom fanin was only a constant - suppose the largest bottom fanin is $c$. We can replace our original formula, $F$ (in our case the formula asserting uniqueness), by a new formula, $F^{\prime}$, as follows. First, we introduce (polynomially many) new variables, one associated with each conjunction of at most $c$ literals. The new uniqueness formula $F^{\prime}$ asserts that $G$ implies $F$, where $G$ is a conjunction of new clauses (called "extension axioms") asserting that each new variable is equivalent to its associated conjunction. The new formula $F^{\prime}$ is still of polynomial size, and furthermore it is a tautology if and only if $F$ is a tautology. It is a standard argument to show that if $F$ has a PK proof where all formulas in the proof are $\Delta_{k}^{+}$, then $F^{\prime}$ has a PK proof where all formulas in the proof are $\Delta_{k}$. To complete the proof of Theorem 13, we replace our original uniqueness formula, $F$, by the new uniqueness formula, $F^{\prime}$. Since $F$ has efficient $\Sigma_{3}^{+}$-Frege proofs, $F^{\prime}$ has efficient $\Sigma_{3}$-Frege proofs.

\section{Consequences and Open Problems}

Tightness of our Result. In this paper, we have shown that if depth-2 Frege systems plus the Integer-Valued Graph Ordering Principle (IGOP) is automatizable, then SSGs are in P. This can be viewed as either an "easyness" result for SSGs or a hardness result for automatizability but more importantly this paper and [4] open up new ways of approaching both problems. Of course, any result in the converse direction would further strengthen this connection. An interesting question is whether there exists an efficient depth-2 proof of uniqueness for SSGs (this would follow immediately if IGOP could be proven in depth-2). However, we conjecture that IGOP does not have efficient depth-2 proofs. Our conjecture is based on the fact that this principle is simply an instance of the usual GOP but where the variables are replaced by $\Delta_{2}$ formulas. GOP is a well-known propositional tautology as it is the classic (and only) example of a family of formulas that have efficient resolution proofs, but that require large width $[9,18]$. That is, it is expressible by small-width clauses, and provable efficiently, but any such efficient proof must raise the depth by 1 (i.e. go from small clauses to large clauses). Our conjecture just says that this phenomena continues to hold when we scale GOP up to higher depth. In fact we make the stronger conjecture that our depth-3 proof of Uniqueness for SSGs is tight. Moreover, we conjecture that the depth-2 proofs of totality for mean payoff games [4] are also tight:

Conjecture 24 (1)Uniqueness for SSGs does not have polynomial-size depth-2 Frege proofs; (2)To- 
tality of MPGs does not have polynomial-size depth-1 (resolution) proofs.

If Conjecture (1) is true, it would imply that SSGs cannot be efficiently reduced (in the type-2 setting) to mean payoff games. This follows from the main result of Atserias and Maneva [4], together with a theorem due to Morioka and Buresh-Oppenheim [11] (Theorem 10 in their paper).

More generally, it is interesting to study low-depth, efficient reductions between statements of totality of various game-theoretic problems. Just as we study the relative strength of various search problems, it is natural to consider the relative proof theoretic strength of the underlying principles, by studying efficient reductions between them in standard low-depth propositional proof systems. The study of total search problems in general, and their corresponding proofs of totality are widely studied in proof complexity. Indeed, the strength of weak systems of arithmetic are classified in terms of what kinds of search problems are provably total in the theory. (For every search problem there is a first order formula expressing that the search problem is total, and conversely for every statement of the form $\exists x A(x, y)$, there is a corresponding total search problem.) PLS figures prominently in this research, as it is precisely the class of total functions that are provably total in the theory $T V^{1}$. It would be interesting to expand this work to include the study of statements of totality for various game-theory problems, as well as to study low-depth reductions between such statements. In particular, consider the propositional statements expressing the following principles: (a) totality for SSGs, (b) totality of mean payoff games, (c) the iteration principle (underlying PLS), (d) the pigeonhole principle (underlying PPAD). Which ones can and cannot be reduced in lowdepth to one another? Answers to these questions would likely yield an understanding of the relative strengths of the corresponding search classes: SSG, MPG, PLS, and PPAD.

\section{References}

[1] M. Alekhnovich and A. Razborov. Resolution is not automatizable unless w[p] is tractable. In IEEE Symposium on Foundations of Computer Science, 2001.

[2] D. Andersson and P. B. Miltersen. The complexity of solving stochastic games on graphs. In ISAAC '09: Proceedings of the 20th International Symposium on Algorithms and Computation, pages 112-121. Springer-Verlag, 2009.

[3] A. Atserias and M. Bonet. On the automatizability of resolution and related propositional proof systems. Information and Computation, 189(2):182-201, 2004.

[4] A. Atserias and E. Maneva. Mean-payoff games and propositional proofs. In S. Abramsky, C. Gavoille, C. Kirchner, F. Meyer auf der Heide, and P. Spirakis, editors, Automata, Languages and Programming, volume 6198 of Lecture Notes in Computer Science, pages 102-113. Springer Berlin / Heidelberg, 2010.

[5] H. Bjorklund and S. Vorobyov. Combinatorial structure and randomized subexponential algorithms for infinite games. Theoretical Computer Science, 349:347-360, 2005.

[6] M. Blum, B. Juba, and R. Williams. Non-monotone behaviors in $\mathrm{min} / \mathrm{max} /$ avg circuits and their relationship to simple stochastic games.

[7] M. Bonet, C. Domingo, R. Gavalda, A. Maciel, and T. Pitassi. No feasible interpolation or automatization for $A C^{0}$-Frege proof systems. Manuscript. 1998. 
[8] M. Bonet, T. Pitassi, and R. Raz. On interpolation and automatization for frege systems. SIAM Journal of Computing, 29(6):1939-1967, 2000.

[9] M. L. Bonet and N. Galesi. optimality of size-width tradeoffs for resolution. Computational Complexity, 10:261-276, 2001.

[10] M. L. Bonet, T. Pitassi, and R. Raz. On interpolation and automatization for frege systems. SIAM J. Comput., 29:1939-1967, April 2000.

[11] J. Buresh-Oppenheim and T. Morioka. Relativized np search problems and propositional proof systems. In 19th IEEE Conference on Computational Complexity (CCC), 2004.

[12] X. Chen, X. Deng, and S. H. Teng. Settling the complexity of two-player nash equilibria. Journal of the ACM, 56, 2009.

[13] M. Clegg, J. Edmonds, and R. Impagliazzo. Using the Gröbner basis algorithm to find proofs of unsatisfiability. In Proceedings of the Twenty-Eighth Annual ACM Symposium on Theory of Computing, pages 174-183, Philadelphia, PA, May 1996.

[14] A. Condon. The complexity of stochastic games. Information and Computation, 96:203-224, 1992.

[15] A. Condon. On algorithms for simple stochastic games. Advances in Computational Complexity Theory, DIMACS Series in Discrete Mathematics and Theoretical Computer Science, 13:5173, 1993.

[16] S. A. Cook and R. A. Reckhow. On the lengths of proofs in the propositional calculus. In Conference Record of Sixth Annual ACM Symposium on Theory of Computing, pages 135-148, Seattle, WA, Apr.-May 1974.

[17] C. Daskalakis, P. Goldberg, and C. H. Papadimtriou. The complexity of computing a nash equilibrium. SIAM Journal on Computing, 39:195-259, 2009.

[18] N. Galesi and M. Lauria. Optimality of size-degree tradeoffs for polynomial calculus. ACM Trans. Comput. Logic, 12:4:1-4:22, November 2010.

[19] H. Gimbert and F. Horn. Simple stochastic games with few random vertices are easy to solve. In R. Amadio, editor, Foundations of Software Science and Computational Structures, volume 4962 of Lecture Notes in Computer Science, pages 5-19. Springer Berlin / Heidelberg, 2008.

[20] N. Halman. Simple stochastic games, parity games, mean payoff games and discounted payoff games are all lp-type problems. Algorithmica, 49(1):37-50, 2007.

[21] K. Krajicek and P. Pudlak. Some consequences of cryptographic conjectures for $S_{2}^{1}$ and EF. In D. Leivant, editor, Logic and Computational Complexity, pages 210-220. Springer-Verlag, 1995.

[22] V. Kumar and R. Tripathi. Algorithmic results in simple stochastic games. In Technical Report 855, University of Rochester, 2004. 
[23] W. Ludwig. A subexponential randomized algorithm for the simple stochastic game problem. Inf. Comput., 117:151-155, 1995.

[24] L. S. Shapley. Stochastic games. Proceedings of the National Academy of Sciences U.S.A., 39:1095-1100, 1953.

[25] R. Somla. New algorithms for solving simple stochastic games. Electron. Notes Theor. Comput. Sci., 119(1):51-65, 2005.

\section{A Proofs of Arithmetic Lemmas}

\section{Proof: Lemma 18}

For any $\Delta_{2}$ formula $F\left(x_{1}, x_{2}, \ldots x_{N}, z \ldots\right)$ there exists a short $\Delta_{2}$ proof that

$$
\forall i x_{i} \leftrightarrow y_{i} \vdash F\left(x_{1}, x_{2}, \ldots x_{N}, z \ldots\right) \leftrightarrow F\left(y_{1}, y_{2}, \ldots y_{N}, z \ldots\right) .
$$

We can prove this by induction on the depth of $F$. For all constant sized formulas $F$ there exists a constant sized proof of the above statement.

Suppose that $F$ has depth $k$, the top level gate is either an AND or an OR with inputs $G_{1}, \ldots G_{m}$. By the induction hypothesis, we can prove for each $j$ that $G_{j}\left(x_{1}, x_{2}, \ldots x_{N}, z, \ldots\right) \leftrightarrow$ $G_{j}\left(y_{1}, y_{2}, \ldots y_{N}, z \ldots\right)$.

\section{Proof: Lemma 19}

Let $x$ and $y$ be positive integers represented by $N$ variables. For any $i$, the following statements have proofs where all formulas are in $\Delta_{2}$

1. $[x \geq y] \longrightarrow[\max (x, y) \leftrightarrow x]$

2. $[x \geq y] \longrightarrow[\min (x, y) \leftrightarrow y]$

Let $\sigma$ represent the sign bit of $x-y$ and $\mathbf{z}$ be the array $\left(x, \bar{y}, 0^{N-1} 1\right)$. Recall that $\sigma$ is in $\Delta_{2}$

$$
\begin{aligned}
\sigma & \stackrel{\text { def }}{=} \operatorname{MOD}_{2}\left(x_{1} \vee \bar{y}_{1} \vee C^{1}(\mathbf{z}, 1)\right) \\
& \leftrightarrow \operatorname{MOD}_{2}(0,1, C(\mathbf{z}, 1))=\neg C(\mathbf{z}, 1) \\
& \leftrightarrow \bigvee_{j \geq 1} \neg A^{1}(\mathbf{z}, 1,1) \wedge \ldots \wedge \neg A^{1}(\mathbf{z}, 1, j-1) \wedge R^{1}(\mathbf{z}, 1, j)
\end{aligned}
$$

and

$$
\neg \sigma=\bigvee_{j \geq 1} \neg R^{1}(\mathbf{z}, 1,1) \wedge \ldots \wedge \neg R^{1}(\mathbf{z}, 1, j-1) \wedge A^{1}(\mathbf{z}, 1, j)
$$

If $[\max (x, y)]_{i}$ could be written as $x_{i} \wedge \neg \sigma \bigvee y_{i} \wedge \sigma$ then the proof would be trivial. However, as discussed in Appendix 4.5, $[\max (x, y)]_{i}$ is actually written 


$$
\left(x_{i} \wedge f_{1}\right) \vee\left(x_{i} \wedge f_{2}\right) \vee \ldots \vee\left(x_{i} \wedge f_{n}\right) \vee\left(y_{i} \wedge g_{1}\right) \vee\left(y_{i} \wedge g_{2}\right) \vee \ldots \vee\left(y_{i} \wedge g_{n}\right)
$$

Where $f_{i}$ are the formulas $\neg R^{1}(\mathbf{z}, 1,1) \wedge \ldots \wedge \neg R^{1}(\mathbf{z}, 1, j-1) \wedge A^{1}(\mathbf{z}, 1, j)$, representing a carry bit being generated at $i$ and propagated to the sign bit. And the $g_{i}$ are the formulas $\neg A^{1}(\mathbf{z}, 1,1) \wedge$ $\ldots \wedge \neg A^{1}(\mathbf{z}, 1, j-1) \wedge R^{1}(\mathbf{z}, 1, j)$ representing an "absorp" bit at $i$ propagated to the sign bit. Note that $[x \geq y]$ is equivalent to $\bigvee_{i} f_{i}$ and $\neg[x \geq y]$ is equivalent to $\bigvee_{i} g_{i}$.

If we can prove for each $j$ that $f_{j} \longrightarrow \max (x, y)_{i} \leftrightarrow x_{i}$ for all $i$, then by combining these sequents we obtain $[x \geq y] \longrightarrow \max (x, y)_{i} \leftrightarrow x_{i}$ for all $i$.

In the easy direction $f_{j} \longrightarrow x_{i} \rightarrow \max (x, y)_{i}$

$$
\begin{aligned}
x_{i} & \longrightarrow x_{i} \wedge f_{j} \\
& \longrightarrow \max (x, y)_{i}
\end{aligned}
$$

To show that $\max (x, y)_{i} \rightarrow x_{i}$ we first need to prove that $f_{j} \longrightarrow \neg g_{k}$ for all $k$.

$$
\begin{aligned}
& A(j, \mathbf{z}) \longrightarrow \neg(\neg A(1, \mathbf{z}) \wedge \ldots \wedge \neg A(k-1, \mathbf{z}) \wedge R(k, \mathbf{z})) \text { for all } k>j \\
& \neg R(k, \mathbf{z}) \longrightarrow \neg(\neg A(1, \mathbf{z}) \wedge \ldots \wedge \neg A(k-1, \mathbf{z}) \wedge R(k, \mathbf{z})) \text { for all } k \leq j
\end{aligned}
$$

From here we can conclude

$$
\begin{aligned}
f_{j} & \longrightarrow \neg g_{k} \\
f_{j} & \longrightarrow \neg\left(g_{k} \wedge y_{i}\right) \\
\neg x_{i}, f_{j} & \longrightarrow \neg \max (x, y)_{i} \\
\max (x, y)_{i}, f_{j} & \longrightarrow x_{i}
\end{aligned}
$$

If $[x \geq y]$ then $f_{j}$ is true for some $j$ and from the above we can derive that $x_{i} \leftrightarrow \max (x, y)_{i}$ for every $i$. The proof for $\min (x, y)$ follows the same structure.

Proof: Lemma 20 There exists a depth 2 polynomial size proof of

$$
[S U M(a, b) \leftrightarrow c] \longrightarrow[S U M(\mathbf{x}, a, b) \leftrightarrow S U M(\mathbf{x}, c)]
$$

Essentially, Lemma 20 states that we can perform nested additions without an increase in depth. The proof proceeds with the same structure as that of Lemma 8.2 in [4]. Atserias and Maneva prove that given $a+b=c$ if the sum $\mathbf{x}+a+b$ generates an overflow then so does the sum $\mathbf{x}+c$. We extend this result to prove that for all $i$ the output bits generated in the two sums are the same.

Let $a, b$, and $c$, be $N$ bit integers $a=a_{1} \ldots a_{N}, b=b_{1} \ldots b_{N}, c=c_{1} \ldots c_{N}$ such that $a+b=c$ and denote $d_{p}$ the bit of carry entering $p$ in the sum $a+b$. Recall that $d_{p}=\operatorname{MAJ}\left(a_{p+1}, b_{p+1}, d_{p+1}\right)$ and $c_{p}=M O D_{2}\left(a_{p}, b_{p}, d_{p}\right)$. 
Define three bitstrings

$$
\begin{aligned}
& \alpha_{p}=a_{1} \ldots a_{p-1} a_{p} c_{p+1} \ldots c_{N} \\
& \beta_{p}=b_{1} \ldots b_{p-1} b_{p} \quad 0 \ldots 0 \\
& \delta_{p}=0 \ldots \quad 0 d_{p} \quad 0 \ldots \quad 0
\end{aligned}
$$

Note that for every $p, \alpha_{p}+\beta_{p}+\delta_{p}=c=a+b$. We will prove that for all $p$ that

$$
\left[S U M\left(\mathbf{x}, \alpha_{P}, \beta_{p} \delta_{p}\right) \leftrightarrow S U M\left(\mathbf{x}, \alpha_{p-1}, \beta_{p-1}, \delta_{p-1}\right)\right]
$$

When $p=N, \alpha_{N}=a, \beta_{N}=b$ and $\delta_{N}=0$ hence $\left[S U M\left(\mathbf{x}, \alpha_{N}, \beta_{N}, \delta_{N}\right) \leftrightarrow S U M(\mathbf{x}, a, b, 0) \leftrightarrow\right.$ $\left.S U M_{i}(\mathbf{x}, a, b)\right]$. And for $p=0, \alpha_{0}=c, \beta_{0}=\delta_{0}=0$ so $\left[S U M_{i}\left(\mathbf{x}, \alpha_{0}, \beta_{0}, \delta_{0}\right) \leftrightarrow S U M_{i}(\mathbf{x}, c, 0,0) \leftrightarrow\right.$ $S U M_{i}(\mathbf{x}, c)$ ]. Both the above formulas can be easily proved using the substitution Lemma 18 and the fac that $[S U M(\mathbf{x}, 0) \leftrightarrow S U M(\mathbf{x})]$. Thus if we can prove (1) for every p then by chaining them together we achieve the result desired.

For simplicity of notation let $\mathbf{z}=\left(\mathbf{z}, \alpha_{p}, \beta_{p}, \delta_{p}\right), \mathbf{z}^{\prime}=\left(\mathbf{x}, \alpha_{p-1}, \beta_{p-1}, \delta_{p-1}\right), s_{i}$ denote $S U M(\mathbf{z})_{i}$, and $s_{i}^{\prime}$ denote $S U M\left(\mathbf{z}^{\prime}\right)_{i}$. Recall that $\mathbf{z}_{i}$ is the sum of the $i^{\text {th }}$ column of $\mathbf{z}$.

It is straightforward to prove that $s_{i} \leftrightarrow s_{i}^{\prime}$ for $i \geq p$. The formula $\left[C^{d}(\mathbf{x}, i)\right]$ depends only on bits $i+1, \ldots, N$ and on those bits $\mathbf{z}$ and $\mathbf{z}^{\prime}$ are identical and so $\left[C^{d}(\mathbf{z}, i)\right] \leftrightarrow\left[C^{d}\left(\mathbf{z}^{\prime}, i\right)\right]$ trivially for all $i \geq p$. Likewise $\left[s_{i} \leftrightarrow s_{i}^{\prime}\right]$ for all $i>p$.

At $i=p$, the $s_{p}$ is the $M O D_{2}$ of $a_{p}, b_{p}, d_{p}$ and the $k$ carry bits $C^{1}(\mathbf{z}, p), \ldots, C^{k}(\mathbf{z}, p)$ where $k$ is the total number of summands in $\mathbf{x}$ plus three. Since all carry bits entering $i$ are the same between $\mathbf{z}$ and $\mathbf{z}^{\prime} s_{p}^{\prime}$ is the $M O D_{2}$ of the same carry bits $C^{1}(\mathbf{z}, p), \ldots, C^{k}(\mathbf{z}, p)$ and $c_{p}$. Additionally $c=a+b$ implies $c_{p}=M O D_{2}\left(a_{p}, b_{p}, d_{p}\right)$. There are only a constant number of bits involved in a proof $\mathrm{MOD}_{2}\left(a_{p}, b_{p}, d_{p}, C^{1}(\mathbf{z}, p), \ldots, C^{k}(\mathbf{z}, p)\right) \leftrightarrow\left(c_{p}, C^{1}(\mathbf{z}, p), \ldots, C^{k}(\mathbf{z}, p)\right)$ so there exists a short $\Delta_{2}^{+}$proof of $s_{p} \leftrightarrow s_{p}^{\prime}$.

When $i<p$, we split into two cases on the value $d_{p-1}$.

Case $d_{p-1}=0$ (easy case):

$$
\begin{aligned}
& \alpha_{p}=a_{1} \ldots a_{p-1} a_{p} c_{p+1} \ldots c_{N} \\
& \beta_{p}=b_{1} \ldots b_{p-1} b_{p} 0 \\
& \delta_{p}=0 \quad \ldots 0 \quad \ldots 0
\end{aligned}
$$

$$
\begin{aligned}
& \alpha_{p-1}=a_{1} \ldots a_{p-1} c_{p} c_{p+1} \ldots c_{N} \\
& \beta_{p-1}=b_{1} \ldots b_{p-1} \quad 00 \quad \ldots 0 \\
& \delta_{p-1}=0 \quad \ldots 0 \quad 00 \quad \ldots 0
\end{aligned}
$$

If $d_{p-1}=0$, at most one of $a_{p}, b_{p}$, and $d_{p}$ is 1 and $c_{p}=M O D_{2}\left(a_{p}, b_{p}, d_{p}\right)=a_{p}+b_{p}+c_{p}$. For every $j, \mathbf{z}_{j}$ and $\mathbf{z}_{j}^{\prime}$, the bitwise column sums are equal. If we can prove that $\left[C^{d}(\mathbf{z}, i) \leftrightarrow C^{d}\left(\mathbf{z}^{\prime}, i\right)\right]$ for all $d$ then we are done.

Each $S(), R(), A()$ formula as defined in Sect. 4 depended only on the bitwise sum, as long as $\mathbf{z}_{j}=\mathbf{z}_{j}^{\prime}, A^{d}(\mathbf{z}, i, j) \leftrightarrow A^{d}\left(\mathbf{z}^{\prime}, i, j\right)$ is a tautology and has a constant size proof. The same statement holds for $R^{d}(\mathbf{z}, i, j)$ and $R^{d}\left(\mathbf{z}^{\prime}, i, j\right)$.

Let $\left[C^{d}(\mathbf{z}, i, j)\right]$ denote $\left[A^{d}(\mathbf{z}, i, j)\right] \wedge \neg\left[R^{d}(\mathbf{z}, i, j-1)\right] \wedge \ldots \wedge \neg\left[R^{d}\left(\mathbf{z}, i, i_{1}\right)\right], i$ receives $d$ bits of carry generated at $j$. Given the above we can prove that for any $j,\left[C^{d}(\mathbf{z}, i, j) \leftrightarrow C^{d}\left(\mathbf{z}^{\prime}, i, j\right)\right]$ 


$$
\begin{aligned}
& \longrightarrow C^{d}(\mathbf{z}, i, j) C^{d}\left(\mathbf{z}^{\prime}, i, j\right) \\
C^{d}(\mathbf{z}, i) & \longrightarrow C^{d}(\mathbf{z}, i, 1), C^{d}(\mathbf{z}, i, 1), \ldots, C^{d}(\mathbf{z}, i, N) \\
C^{d}(\mathbf{z}, i) & \longrightarrow C^{d}\left(\mathbf{z}^{\prime}, i\right)
\end{aligned}
$$

and of course the other direction holds as well so for all $i<p, C^{d}(\mathbf{z}, i) \leftrightarrow C^{d}\left(\mathbf{z}^{\prime}, i\right)$ whenever $d_{p-1}=1$. Since all bits of $\mathbf{z}$ and $\mathbf{z}^{\prime}$ are equivalent when $i<p$ this is sufficient to prove $s_{i} \leftrightarrow s_{i}^{\prime}$.

Case $d_{p-1}=1$ :

When $d_{p-1}=1$, at least two of $a_{b}, b_{p}$, and $d_{p}$ are 1 and $c_{p}=a_{p}+b_{p}+d_{p}-2$ so the picture becomes:

$$
\begin{array}{llll}
\alpha_{p}=a_{1} \ldots a_{p-1} c_{p} c_{p+1} \ldots c_{N} & \alpha_{p-1}=a_{1} \ldots a_{p-2} c_{p-1} c_{p} \ldots c_{N} \\
\beta_{p}=b_{1} \ldots b_{p-1} & 10 & \ldots 0 & \beta_{p-1}=b_{1} \ldots b_{p-2} b_{p-1} 0 \ldots 0 \\
\delta_{p}=0 \quad \ldots 0 & 10 & \ldots 0 & \delta_{p-1}=0 \ldots 0
\end{array}
$$

At the $p^{t h}$ column on the left hand side $a_{p}, b_{p}, d_{p}$ has been replaced by the equivalent bits $c_{p}, 1,1$ without affecting the column sums. At bit $p-1, \mathbf{z}_{p-1}^{\prime}=\mathbf{z}_{p-1}+1$ and at all $i<p-1, \mathbf{z}_{i}=\mathbf{z}_{i}^{\prime}$. In order to prove $s_{i} \leftrightarrow s_{i}^{\prime}$ for $i<p$ we need to prove

$$
\begin{gathered}
C^{d}(\mathbf{z}, i) \leftrightarrow C^{d}\left(\mathbf{z}^{\prime}, i\right) \text { for all } i<p-1 \text { and all } d, \text { and } \\
C^{d}(\mathbf{z}, p-1) \leftrightarrow C^{d-1}\left(\mathbf{z}^{\prime}, p-1\right)
\end{gathered}
$$

(3) means there is exactly one less carry into $\mathbf{z}^{\prime}$ at index $p-1$. This makes up for the parity difference between $\mathbf{z}_{p-1}$ and $\mathbf{z}_{p-1}^{\prime}$. To prove (2) we will first show that for all $j \neq p-1$ and $i<p-1$

$$
\left[A(\mathbf{z}, i, j) \leftrightarrow A\left(\mathbf{z}^{\prime}, i, j\right)\right], \text { and }\left[\neg R(\mathbf{z}, i, j) \leftrightarrow \neg R\left(\mathbf{z}^{\prime}, i, j\right)\right]
$$

Suppose that $i<p-1$, if $j \neq p-1 . S^{d}(\mathbf{z}, i, j)=S^{d}\left(\mathbf{z}^{\prime}, i, j\right)$ whenever the $\ell$-window used to compute $S()$ does not include either bit $p-1$ or bit $p$. On the other hand

$$
2^{m}\left(a_{p-1}+b_{p-1}+\mathbf{x}_{p-1}\right)+2^{m-1}\left(2+c_{p}+\mathbf{x}_{p}\right)=2^{m}\left(a_{p-1}+b_{p-1}+1+\mathbf{x}_{p-1}\right)+2^{m-1}\left(c_{p}+\mathbf{x}_{p}\right)
$$

so as long as the $\ell$-window contains both bit $p-1$ and $p$ the state computations over $\mathbf{z}$ and $\mathbf{z}^{\prime}$ are still equivalent. The only index where the $\ell$-window holds exactly one of $\{p, p-1\}$ is when $j=p+\ell$

$$
\begin{aligned}
S^{d}\left(\mathbf{z}^{\prime}, i, p+\ell\right) & =\left(2^{\ell-1}\left(\mathbf{z}_{p}-2\right)+\ldots+2^{0} \mathbf{z}_{p+\ell}\right) \bmod 2^{\ell}-2^{\ell} \\
& =\left(-2^{\ell}+2^{\ell-1} \mathbf{z}_{p}+\ldots+2^{0} \mathbf{z}_{p+\ell}\right) \bmod 2^{\ell}-2^{\ell} \\
& =S(\mathbf{z}, p+\ell, j)
\end{aligned}
$$

As long as the column sums $\mathbf{z}_{j}$ and $\mathbf{z}_{j}^{\prime}$ are equal then the equality above is enough to prove (4). 
The only interesting case to consider are when $j=p$.

$$
\begin{aligned}
2 \cdot S(\mathbf{z}, i, p-1)+a_{p}+b_{p}+d_{p}= & 2 \cdot\left[2^{\ell-1} \mathbf{z}_{p-1-\ell}^{\prime}+\ldots+2^{0}\left(\mathbf{z}_{p-1}^{\prime}-1\right) \bmod 2^{\ell}-2^{\ell}\right] \\
& +a_{p}+b_{p}+d_{p} \\
= & 2 \cdot\left[S\left(\mathbf{z}^{\prime}, i, p-1\right)-1\right]+a_{p}+b_{p}+d_{p} \\
= & 2 \cdot\left[S\left(\mathbf{z}^{\prime}, i, p-1\right)\right]+a_{p}+b_{p}+d_{p}-2 \\
= & 2 \cdot\left[S\left(\mathbf{z}^{\prime}, i, p-1\right)\right]+c_{p}
\end{aligned}
$$

So (4) also holds for $j=p$. The last index to consider is $j=p-1$ it is in general not true that $A^{d}(\mathbf{z}, i, j) \leftrightarrow A^{d}\left(\mathbf{z}^{\prime}, i, i\right)$ but we will prove that if there is an Accept in one of $\mathbf{z}$ or $\mathbf{z}^{\prime}$ there is a corresponding accept "close to" $j$ in the other array. Let $j=p-1$,

$$
\begin{aligned}
A(\mathbf{z}, i, p-1) & \rightarrow 2 \cdot S(\mathbf{z}, i, p-2)+a_{p-1}+b_{p-1}+\mathbf{x}_{p-1} \geq 0 \\
& \rightarrow 2 \cdot S\left(\mathbf{z}^{\prime}, i, p-2\right)+a_{p-1}+b_{p-1}+1+\mathbf{x}_{p-1} \geq 0 \\
& \rightarrow A\left(\mathbf{z}^{\prime}, i, p-1\right)
\end{aligned}
$$

By the same reasoning, $\neg R(\mathbf{z}, i, p-1) \rightarrow \neg R\left(\mathbf{z}^{\prime}, i, p-1\right)$. The reverse direction does not hold if $C^{d}\left(\mathbf{z}^{\prime}, i\right)$ accepts at $p-1$ and $C^{d}(\mathbf{z}, i)$ doesn't, or if $C^{d}(\mathbf{z}, i)$ rejects at $p-1$ and $C^{d}\left(\mathbf{z}^{\prime}, i\right)$ does not. It will be convenient to define here $N(\mathbf{z}, i, j)=2 \cdot S(\mathbf{z}, i-1, j)+\mathbf{z}_{i} \cdot A(\mathbf{z}, i, j)$ and $R(\mathbf{z}, i, j)$ are exactly equal to $N(\mathbf{z}, i, j) \geq 0$ and $N(\mathbf{z}, i, j) \leq-k$ respectively.

$$
\begin{aligned}
N\left(\mathbf{z}^{\prime}, i, p-1\right) & =2 \cdot S\left(\mathbf{z}^{\prime}, i, p-2\right)+\left(a_{p-1}+b_{p-1}+1\right)+\mathbf{x}_{p-1} \\
& =2 \cdot S(\mathbf{z}, i, p-2)+a_{p-1}+b_{p-1}+1+\mathbf{x}_{p-1} \\
& =N(\mathbf{z}, i, p-1)+1
\end{aligned}
$$

Suppose that $\neg R\left(\mathbf{z}^{\prime}, i, p-1\right)$, as long as $N\left(\mathbf{z}^{\prime}, p-1\right)>-k+1$ we are in good shape. Suppose for contradiction that $N\left(\mathbf{z}^{\prime}, i, p-1\right) \leq-k+1$.

$$
\begin{aligned}
N\left(\mathbf{z}^{\prime}, i, p\right) & \leq 2 \cdot(-k+1)+c_{p}+\mathbf{x}_{p} \\
& \leq-2 k+2+1+k-3 \\
& \leq-k-2
\end{aligned}
$$

and since we know $\neg R\left(\mathbf{z}^{\prime}, i, p\right)$, it cannot be the case that $N\left(\mathbf{z}^{\prime}, i, p-1\right) \leq-k+1$. We've proved by contradiction $\neg R\left(\mathbf{z}^{\prime}, i, p-1\right) \rightarrow \neg R(\mathbf{z}, i, p-1)$.

Next we show that if that $A^{d}\left(\mathbf{z}^{\prime}, i, p-1\right)$ implies there is a corresponding accept in $C^{d}(\mathbf{z}, i)$. As above we know that $N\left(\mathbf{z}^{\prime}, i, p-1\right)=N(\mathbf{z}, i, p-1)+1$. The only case we need to be concerned with is when $N\left(\mathbf{z}^{\prime}, i, p-1\right)=0$ and $N(\mathbf{z}, i, p-1)=-1$. If this is the case

$$
\begin{aligned}
N(\mathbf{z}, i, p) & =2 \cdot S(\mathbf{z}, i, p-1)+\left(a_{p}+b_{p}+d_{p}\right)+\mathbf{x}_{p} \\
& \geq-2+a_{p}+b_{p}+d_{p} \geq 0
\end{aligned}
$$

since at least two of $a_{p}, b_{p}$, and $d_{p}$ are $1\left(\right.$ from $\left.d_{p-1}=1\right)$.

To summarize what we can prove so far, for $i<p-1$, for all $d$ 


$$
\begin{aligned}
A^{d}(\mathbf{z}, i, j) & \leftrightarrow A^{d}\left(\mathbf{z}^{\prime}, i, j\right) \text { when } j>p-1 \\
\neg R^{d}(\mathbf{z}, i, j) & \leftrightarrow \neg R^{d}\left(\mathbf{z}^{\prime}, i, j\right) \text { when } j>p-1 \\
A^{d}(\mathbf{z}, i, p-1) & \rightarrow A^{d}\left(\mathbf{z}^{\prime}, i, p-1\right) \\
\neg R^{d}(\mathbf{z}, i, p-1) & \rightarrow \neg R^{d}\left(\mathbf{z}^{\prime}, i, p-1\right) \\
A^{d}\left(\mathbf{z}^{\prime}, i, p-1\right) & \leftarrow A^{d}(\mathbf{z}, i, p-1) \vee A^{d}(\mathbf{z}, i, p) \\
\neg R^{d}\left(\mathbf{z}^{\prime}, i, p-1\right) & \leftarrow \neg R^{d}(\mathbf{z}, i, p-1)
\end{aligned}
$$

Which is sufficient to prove $\left[C^{d}(\mathbf{z}, i) \leftrightarrow C^{d}\left(\mathbf{z}^{\prime}, i\right)\right]$ for $i<p-1$.

Finally we consider the carries at $i=p-1$. We want to prove (3) that at $i=p-1, C^{d}(\mathbf{z}, p-$ $1, j) \leftrightarrow C^{d-1}\left(\mathbf{z}^{\prime}, p-1, j\right)$

$\begin{array}{cccc}c_{p} c_{p+1} \ldots c_{N} & c_{p} c_{p+1} \ldots c_{N} \\ 10 & \ldots 0 & 00 & \ldots 0 \\ 10 & \ldots 0 & 00 & \ldots 0\end{array}$

Recall that calculating $C^{d}(\mathbf{z}, p-1)$ is equivalent to starting the circuit in state $-d$. If $i+1<$ $j \leq i+\ell$

$$
\begin{aligned}
S^{d}(\mathbf{z}, i, j)= & -d \cdot 2^{j-i}+\left(2+c_{p}+\mathbf{x}_{p}\right) 2^{j-i-1}+\ldots+\mathbf{z}_{j} 2^{0} \\
= & -d \cdot 2^{j-i}+2^{j-i}+\left(c_{p}+\mathbf{x}_{p}\right) 2^{j-i-1}+\ldots+\mathbf{z}_{j} 2^{0} \\
= & (-d+1) 2^{j-i}+\left(c_{p}+\mathbf{x}_{p}\right) 2^{j-i-1}+\ldots+\mathbf{z}_{j} 2^{0} \\
= & S^{d-1}\left(\mathbf{z}^{\prime}, i, j\right) \\
& N^{d}(\mathbf{z}, i, j)=S^{d}(\mathbf{z}, i, j) \cdot 2+\mathbf{z}_{j} \\
= & S^{d-1}\left(\mathbf{z}^{\prime}, i, j\right)+\mathbf{z}_{j}^{\prime} \\
= & N^{d-1}\left(\mathbf{z}^{\prime}, i, j\right)
\end{aligned}
$$

At $j=i+1$

$$
\begin{aligned}
N^{d}(\mathbf{z}, i, j) & =-d \cdot 2+2+c_{p}+\mathbf{x}_{p} \\
& =-(d-1) \cdot 2+c_{p}+\mathbf{x}_{p} \\
& =N^{d-1}\left(\mathbf{z}^{\prime}, i, j\right)
\end{aligned}
$$

and of course the remaining $\ell$ windows are unaffected by the $-d$ and are identical in $\mathbf{z}$ and $\mathbf{z}^{\prime}$ so of course $N^{d}(\mathbf{z}, i, j)=N^{d-1}(\mathbf{z}, i, j)=N^{d-1}\left(\mathbf{z}^{\prime}, i, j\right)$ whenever $j>i+\ell$.

We have shown that for any $a, b$ and $c=a+b$ there exists a $P K$-proof where all formulas are in $\Delta_{2}$ of $S U M(\mathbf{x}, a, b) \leftrightarrow S U M(\mathbf{x}, c)$. It does not immediately follow that from $a-b=c$ we can derive the same equality. (Recall that $a-b$ is actually the sum of three terms $a, \bar{b}, 1$.) However we can overcome this difficulty with one more trick.

$\operatorname{SUM}(a, b, 1) \leftrightarrow S U M(a 1, \bar{b} 1)$ where $a 1$ and $\bar{b} 1$ are the strings $a$ and $\bar{b}$ appended with a 1 on the right. Then certainly $a 1+\bar{b} 1=c$ and we can apply the same proof as above to obtain $\operatorname{SUM}(a 1, \bar{b} 1, \mathbf{x}) \leftrightarrow S U M(c, \mathbf{x})$. 


\section{Proof: Corollary 21}

$$
\left[x+a+b \leftrightarrow 1^{N}\right] \longrightarrow[S U M(a, b) \leftrightarrow \bar{x}]
$$

For any $x$ it is not hard to prove that $\operatorname{SUM}(x, \bar{x})=1^{N}$. Given that $\operatorname{SUM}(x, a, b)=1^{N}$, we will prove that if $\bar{x}$ is not equal to $\operatorname{SUM}(a, b)$ then at some $i \operatorname{SUM}(x, \bar{x})$ is not equal to $1^{N}$.

Suppose that $\bar{x} \neq S U M(a, b)$, and let $p$ be the last (least significant) index where they differ. Let $d$ be the carry bit into $p$ in the $S U M(a, b)$. Consider the strings, $\alpha_{p}, \beta_{p}, \delta_{p}$

$$
\begin{aligned}
& \alpha_{p}=a_{1} \ldots a_{p-1} a_{p} \bar{x}_{p+1} \ldots \bar{x}_{N} \\
& \beta_{p}=b_{1} \ldots b_{p-1} b_{p} \quad 0 \ldots \quad 0 \\
& \delta_{p}=0 \ldots \quad 0 d \quad 0 \ldots \quad 0
\end{aligned}
$$

From the proof of Lemma 20 we have that $\left[S U M(x, a, b) \leftrightarrow S U M\left(x, \alpha_{p}, \beta_{p}, \delta_{p}\right)\right]$ and additionally all carry bits going into bit $p$ are the same for both sums. Observe that the carry bits going into bit $p$ are also equal to the carry bits going into $p$ in the sum $\operatorname{SUM}(x, \bar{x})$.

However, we chose $p$ so that $\operatorname{MOD}_{2}\left(a_{p}, b_{p}, d\right) \neq \bar{x}_{p}$ so at bit $p, \operatorname{SUM}(x, \bar{x})$ is not equal to $S U M(x, \alpha, \beta, \delta)_{p}=S U M(x, a, b)_{p}=1$.

In particular when $[x \leftrightarrow a-b]$, if we can show that $\left[S U M(x, \bar{a}, b) \leftrightarrow 1^{N}\right]$ then $[\bar{x} \leftrightarrow S U M(\bar{a}, b)]$.

$$
\begin{aligned}
\operatorname{SUM}(x, \bar{a}, b) & \leftrightarrow \operatorname{SUM}\left(a, \bar{b}, 0^{N-1} 1, \bar{a}, b\right) \\
\operatorname{SUM}(x, \bar{a}, b) & \leftrightarrow \operatorname{SUM}(\bar{b}, b) \leftrightarrow 1^{N} \\
\bar{x} & \leftrightarrow \operatorname{SUM}(\bar{a}, b)
\end{aligned}
$$

And when $[x \leftrightarrow a+b]$,

$$
\begin{aligned}
\operatorname{SUM}\left(x, \bar{a}, \bar{b}, 0^{N-1} 1\right) & \leftrightarrow \operatorname{SUM}\left(a, b, \bar{a}, \bar{b}, 0^{N-1} 1\right) \\
& \leftrightarrow \operatorname{SUM}(\bar{b}, b) \leftrightarrow 1^{N} \\
\bar{x} & \leftrightarrow \operatorname{SUM}\left(\bar{a}, \bar{b}, 0^{N-1} 1\right)
\end{aligned}
$$

\section{Proof: Lemma 22}

Let $x, y, x^{\prime}, y^{\prime}$ be N-bit integers padded by $k$ sign bits where $k \geq 4$. Then there exists a $\Delta_{2}$ proof for

$$
\left[x>x^{\prime}\right],\left[x=y-\frac{1}{2^{m}} y\right],\left[x^{\prime}=y^{\prime}-\frac{1}{2^{m}} y^{\prime}\right] \longrightarrow\left[x-x^{\prime}-y+y^{\prime}<0\right]
$$

First we will prove that $\left[y^{\prime}<y\right]$ by contradiction. $\left[y^{\prime} \geq y\right],\left[x=y-\frac{1}{2^{m}} y\right],\left[x^{\prime}=y^{\prime}-\right.$ $\left.\frac{1}{2^{m}} y^{\prime}\right] \longrightarrow\left[x^{\prime} \geq x\right]$. Using Lemma 20 we can rewrite the sum $\left[x^{\prime}-x\right]$ in terms of the $y$ and $y^{\prime}$ variables. Let $\mathbf{z}$ be the following array 


$$
\begin{aligned}
& y_{1}^{\prime} y_{2}^{\prime} \ldots y_{m-1}^{\prime} y_{m}^{\prime} \ldots \quad y_{N}^{\prime} \\
& \overline{y_{1} y_{2}} \ldots \overline{y_{m-1} y_{m}} \ldots \quad \overline{y_{N}} \\
& 11 \ldots{\overline{y^{\prime}}}_{1} \quad{\overline{y^{\prime}}}_{2} \ldots{\overline{y^{\prime}}}_{N-m} \\
& 00 \ldots y_{1} \quad y_{2} \ldots y_{N-m} \\
& 00 \ldots 0 \quad 0 \ldots \quad 1 \\
& 00 \ldots 0 \quad 0 \ldots \quad 1
\end{aligned}
$$

Then $\left[S U M(\mathbf{z})=x^{\prime}-x\right]$ and we want to show that $S U M(\mathbf{z}) \geq 0$.

This follows from looking at the carry circuit for these $\mathbf{z}$. Clearly no more than two carries can enter the sign bit. The bitwise sum in the first $k$ bits is exactly 2 , this follows from the fact that positive numbers lead with 0 and both $y$ and $y^{\prime}$ are padded by $k$ sign bits. Examining the carry circuit for 6 numbers we can see that this leads to a reject within the first $k$ bits.

If we can show that at least 2 bits of carry enter the sign bit then because $2+y_{1}^{\prime}+\bar{y}_{1}+1=4=$ $0 \mathrm{MOD}_{2}$ we have shown that $\left[S U M(\mathbf{z})=x^{\prime}-x\right]$ is positive. Suppose that $y^{\prime}-y \geq 0$, then there is one bit of carry entering the sign bit in the $S U M\left(y^{\prime}, \bar{y}\right)$ and the following formula is true

$$
\bigvee_{p>1}\left[\neg R^{1}(\mathbf{y}, 1,1) \wedge \ldots \wedge \neg R(\mathbf{y}, 1, p-1) \wedge A^{1}(\mathbf{y}, 1, p)\right]
$$

where $\mathbf{y}$ is the array $\left(y^{\prime}, \bar{y}\right)$. In other words, there is some index $p$ where both $y_{p}^{\prime}$ and $\bar{y}_{p}$ are true and for all $i<p$ at least one of $y_{i}^{\prime}$ and $\bar{y}_{i}$ are true. This is enough information to compute the column sums $\mathbf{z}_{i}$ and the formula $C^{2}(\mathbf{z}, 1)$. For all $i, \mathbf{z}_{i} \geq y_{i}^{\prime}+\bar{y}_{i}+{\overline{y^{\prime}}}_{i-m}+y_{i-m}$

$$
\mathbf{z}_{i} \geq \begin{cases}2 & \text { if } i<p \\ 3 & \text { if } i=p \\ 1 & \text { if } p<i<p+m \\ 2 & \text { if } i=N \text { or } i=p+m, p+m \leq N\end{cases}
$$

This is enough to generate two carries into the $\operatorname{sign}$ bit. At $i=\min (p+m, N)$ the formula below is true

$$
C^{2}(\mathbf{z}, 1, i)=\neg R^{2}(\mathbf{z}, 1,1) \wedge \ldots \wedge \neg R^{2}(\mathbf{z}, 1, i-1) \wedge A^{2}(\mathbf{z}, 1, i)
$$

Putting it all together,

$$
\begin{aligned}
{\left[y^{\prime} \geq y\right],\left[x=y-\frac{1}{2^{m}} y\right],\left[x^{\prime}=y^{\prime}-\frac{1}{2^{m}} y^{\prime}\right] } & \longrightarrow\left[x^{\prime} \geq x\right] \\
\neg\left[x^{\prime} \geq x\right],\left[x=y-\frac{1}{2^{m}} y\right],\left[x^{\prime}=y^{\prime}-\frac{1}{2^{m}} y^{\prime}\right] & \longrightarrow \neg\left[y^{\prime} \geq y\right] \\
{\left[x>x^{\prime}\right],\left[x=y-\frac{1}{2^{m}} y\right],\left[x^{\prime}=y^{\prime}-\frac{1}{2^{m}} y^{\prime}\right] } & \longrightarrow\left[y>y^{\prime}\right] \\
{\left[y>y^{\prime}\right] } & \longrightarrow\left[\frac{1}{2^{m}} y>\frac{1}{2^{m}} y^{\prime}\right] \\
{\left[x>x^{\prime}\right],\left[x=y-\frac{1}{2^{m}} y\right],\left[x^{\prime}=y^{\prime}-\frac{1}{2^{m}} y^{\prime}\right] } & \longrightarrow\left[\frac{1}{2^{m}} y>\frac{1}{2^{m}} y^{\prime}\right]
\end{aligned}
$$


And using Lemma 20 we can also prove that

$$
\begin{aligned}
& {\left[x-x^{\prime}-y+y^{\prime} \leftrightarrow y-\frac{1}{2^{m}} y-y^{\prime}+\frac{1}{2^{m}} y^{\prime}-y+y^{\prime}\right]} \\
& {\left[x-x^{\prime}-y+y^{\prime} \leftrightarrow \frac{1}{2^{m}} y^{\prime}-\frac{1}{2^{m}} y\right]}
\end{aligned}
$$

thus

$$
\left[x>x^{\prime}\right],\left[x=y-\frac{1}{2^{m}} y\right],\left[x^{\prime}=y^{\prime}-\frac{1}{2^{m}} y^{\prime}\right] \quad \longrightarrow \quad\left[x-x^{\prime}-y+y^{\prime}<0\right]
$$

\section{Proof: Lemma 23}

We will show that as long as all summands are sufficiently padded there are short proofs of:

(1) The sum of two negative sums is negative.

(2) The sum of two positive sums is positive.

Formally we want to prove the following two sequents in $\Delta_{2}$

$$
\begin{gathered}
S U M(\mathbf{x})_{1}, S U M(\mathbf{y})_{1} \longrightarrow S U M(\mathbf{x}, \mathbf{y})_{1} \\
\neg S U M(\mathbf{x})_{1}, \neg S U M(\mathbf{y})_{1} \longrightarrow \neg S U M(\mathbf{x}, \mathbf{y})_{1}
\end{gathered}
$$

Let $k$ be the number of summands in $S U M(\mathbf{x}, \mathbf{y})$ (all integers are padded by at least $k$ bits) and $s=s_{x}+s_{y}$ be the bitwise sum for each of those $k$ bits. $s_{x}$ and $s_{y}$ respectively are the bitwise sums in $\mathbf{x}$ and $\mathbf{y}$.

Claim 1 For any $\mathbf{z}, C^{m}(\mathbf{z}, 1)$ accepts within the first $k$ bits whenever $m>s_{z}$ and rejects whenever $m<s_{z}$. When $m=s_{z} C^{m}(\mathbf{z})$ does not reject or accept in the first $k$ bits and further $S^{m}(\mathbf{z}, 1, k)=$ $-m$.

Proof:

Recall that determining whether $m$ carries enter the first bit of $\mathbf{z}$ is the same as determining whether 1 carry is generated in bit the first bit of $\mathbf{z}^{\prime}$ where $\mathbf{z}^{\prime}$ is $\mathbf{z}$ prefixed by $2^{\ell}-m$. And in particular $S^{1}\left(\mathbf{z}^{\prime}, 1,1+\ell\right)=S^{m}(\mathbf{z}, 1,1)=-m$.

If for any $i<k, S^{m}(\mathbf{z}, 1, i)>\frac{-s_{z}}{2}$ then at bit $i+1, s_{z}+2 S^{m}(\mathbf{z}, 1,1)>0$ and the carry circuit accepts. Likewise, if $S^{m}(\mathbf{z}, 1, i)<\frac{-s_{z}-k}{2}$ then the carry circuit rejects at bit $i+1$. We will prove by induction that $S^{m}(\mathbf{z}, 1, i)$ decreases by at least 1 for each $i$ when $s<m$ and likewise increases by at least 1 when $s>m$. Then within at most $k$ steps $2 \cdot S^{m}(\mathbf{z}, 1, i)+s$ will reach either the accept or reject state.

Suppose that $S^{m}(\mathbf{z}, 1, i)=-m-c$ and $s<m$, then at $i+1$ the state is exactly $2 \cdot(-m-$ $c)+s=-2 m-2 c+s<-m-c-1$. And if $S^{m}(\mathbf{z}, 1, i)=-m+c$ and $s>m$ then at $i+1$, $S^{m}(\mathbf{z}, 1, i+1)=2 \cdot(-m+c)+s=-2 m+2 c+s>-m+c+1$. 
Given the claim, we know that at least $s-1$ bits of carry enter at bit 1 in $S U M(\mathbf{x}, \mathbf{y})$. If we can show that whenever $S U M(\mathbf{x})$ and $S U M(\mathbf{y})$ are both negative then $C^{s}((\mathbf{x}, \mathbf{y}), 1)$ is false then we are in good shape, since the parity of $s-1+s$ is 1 .

Let $\mathbf{x}^{\prime}$ be the $k \times N$ array containing $\mathbf{x}$ as well as additional rows of zeros and $\mathbf{y}^{\prime}$ be the same for y. Clearly, $S U M(\mathbf{y}) \leftrightarrow S U M\left(\mathbf{y}^{\prime}\right)$ and $S U M(\mathbf{x}) \leftrightarrow S U M\left(\mathbf{x}^{\prime}\right)$ and thus the carry circuits $C^{s_{x}}\left(\mathbf{x}^{\prime}, 1\right)$ and $C^{s_{y}}\left(\mathbf{y}^{\prime}, 1\right)$ both reject. Suppose that the reject occurs first in bit $i$ of $\mathbf{x}^{\prime}$. Then for all $j<i$, $\neg A^{s_{x}}\left(\mathbf{x}^{\prime}, 1, j\right)$ and $\neg A^{s_{y}}\left(\mathbf{y}^{\prime}, 1, j\right)$ and at $i \neg A^{s_{y}}\left(\mathbf{y}^{\prime}, 1, i\right)$ and $R^{s_{x}}\left(\mathbf{x}^{\prime}, 1, i\right)$. We will prove that at bit $i, R^{s}((\mathbf{x}, \mathbf{y}), 1, i)$ and for all $j<i, \neg A((\mathbf{x}, \mathbf{y}), 1, j)$.

From the claim we know that $i>k$ and $\neg A((\mathbf{x}, \mathbf{y}), 1, j)$ for $j<k$. Let $P(j)$ be the formula true if and only if $S^{s}((\mathbf{x}, \mathbf{y}), 1, j)=S^{s_{x}}\left(\mathbf{x}^{\prime}, 1, j\right)+S^{x_{y}}(\mathbf{y}, 1, j)$. Note that $P(j)$ is a constant sized formula. We will show that either $P(j)$ holds for all $k<j<i$ and $R^{s}((\mathbf{x}, \mathbf{y}), 1, i)$ or there exists some $l<i$ such that $P(j)$ holds for all $k<j<l$ and $R^{s}((\mathbf{x}, \mathbf{y}), 1, l)$.

When $j=k$, we have by construction that $S^{s_{x}}\left(\mathbf{x}^{\prime}, 1, k\right)+S^{s_{y}}\left(\mathbf{y}^{\prime}, 1, k\right)=-s_{x}-s_{y}=-s=$ $S^{s}((\mathbf{x}, \mathbf{y}), 1, k)$, so $P(k)$ is true. Suppose that $A^{s}((\mathbf{x}, \mathbf{y}), 1, k+1)$ is true. Then

$$
\begin{aligned}
S^{s}((\mathbf{x}, \mathbf{y}), 1, k)+(\mathbf{x}, \mathbf{y})_{i} & \geq 0 \\
S^{s_{x}}\left(\mathbf{x}^{\prime}, 1, k\right)+S^{s_{y}}\left(\mathbf{y}^{\prime}, 1, k\right)+\mathbf{x}_{i}^{\prime}+\mathbf{y}_{i}^{\prime} & \geq 0
\end{aligned}
$$

where we have substituted for $S^{x}((\mathbf{x}, \mathbf{y}), 1, k)$ becauce $P(k)$ is true. But this would imply that at least one of $A^{s_{x}}\left(\mathbf{x}^{\prime}, 1, k\right)$ of $A^{s_{y}}\left(\mathbf{y}^{\prime}, 1, k\right)$ which is a contradiction. We defined the transitions so that either, either the circuit reached Reject in which case we are done, or at $i=k+1$ then $S^{s_{x}}\left(\mathbf{x}^{\prime}, 1, i\right)=2 \cdot S^{s_{x}}\left(\mathbf{x}^{\prime}, 1, i-1\right)+\mathbf{x}_{i}$ and likewise for $S^{s_{y}}\left(\mathbf{y}^{\prime}, 1, i\right)$ and $S^{s}((\mathbf{x}, \mathbf{y}), 1, i)$ so $P(k+1)$ is also true. Continuing by induction we have that the carry circuit on $(\mathbf{x}, \mathbf{y})$ for $s$ bits rejects between bits $k$ and $i$.

It remains to show that $P(i-1), R^{s_{x}}\left(\mathbf{x}^{\prime}, 1, i\right), \neg A^{s_{y}}\left(\mathbf{y}^{\prime}, 1, i\right) \longrightarrow R^{s}((\mathbf{x}, \mathbf{y}), 1, i)$. As before, this follows immediately from $P(i-1)$ and the fact that $\mathbf{x}_{i}^{\prime}+\mathbf{y}_{i}^{\prime}=(\mathbf{x}, \mathbf{y})_{i}$.

The proof for (2) is very similar. If $s_{x}$ bits of carry are generated for $\mathbf{x}$ and $s_{y}$ bits of carry are generated for $\mathbf{y}$ then the maximum of the two accepting positions also generates $s$ bits of carries for the sum of $(\mathbf{x}, \mathbf{y})$. Let $i$ be the minimum of the two accepting positions and suppose the first accept occurs in $\mathbf{x}$. Then by the argument above, $P(i-1)$ holds. Define $Q(j)$ to be the formula true if and only if $S^{s}((\mathbf{x}, \mathbf{y}), 1, j) \geq S^{s_{y}}(\mathbf{y}, 1, j)$. By the fact that $i$ is an accept for $\mathbf{x}, Q(i)$ holds. We can prove inductively that as long as the circuit has not yet reached a sink, $Q(i) \longrightarrow Q(i+1)$. Let $j$ be the index at which the carry circuit for $\mathbf{y}$ accepts, then at $j, Q(j-1)$ ensures that $A^{s}((\mathbf{x}, \mathbf{y}), 1, j)$ is true. 